\title{
Design and Analysis of Cooperative Broadcast Scheme Based on Reliability in Mesh Network
}

\author{
Zufang Dou (D), Jianwen Tian, Qiaoli Yang, and Liben Yang \\ School of Automation \& Electrical Engineering, Lanzhou JiaoTong University, Lanzhou, China \\ Correspondence should be addressed to Zufang Dou; douzufang@126.com
}

Received 31 January 2021; Revised 4 May 2021; Accepted 24 May 2021; Published 12 June 2021

Academic Editor: Carlos T. Calafate

Copyright (๑) 2021 Zufang Dou et al. This is an open access article distributed under the Creative Commons Attribution License, which permits unrestricted use, distribution, and reproduction in any medium, provided the original work is properly cited.

In order to ensure reliable data transmission of sender, the tree-based network is extended to Mesh network by collaborative technology, and the SW-HBH-CARQ broadcast scheme is introduced for the first time. Then, in order to solve the problems of broadcast storm and energy saving, SW-HBH-oiCARQ, SW-HBH-ieCARQ, and SW-HBH-oieCARQ are further proposed. Then, the four broadcasting schemes are analyzed quantitatively, and the performance indicators such as system energy consumption and time delay are obtained. Finally, the relationships between the performance indexes and the parameters are discussed by numerical simulation. At the same time, the effectiveness of the proposed schemes in energy saving is verified by comparing with the corresponding noncollaborative broadcasting scheme. However, the latency of proposes scheme is greater than uncooperative scheme, which is the cost of saving energy and resolving broadcast storms.

\section{Introduction}

With the rise of emerging new technologies, the intelligent transportation system formed by applying wireless communication technology, sensor technology, Internet of things technology, and artificial intelligence algorithm to the field of transportation, as an important part of smart city, has been widely concerned by academia, industry, and government. As the core technology foundation of it, the vehicular ad hoc network (VANET) plays an important role in optimizing traffic management, automatic driving, avoiding traffic accidents, and other application fields.

The improvement, deployment, and implementation of VANET will make intelligent transportation develop rapidly, and also bring a lot of benefits to people's social lives and works. Compared with the traditional intelligent transportation system (ITS), VANET puts more emphasis on the transmission of data information among various nodes in the network, such as vehicle units, roadside units, and pedestrians. In particular, the application of safety is the most basic and important application of VANET, whose goal is to reduce the probability of traffic accidents and avoid secondary injuries [1]. Safety messages can be divided into many kinds. According to the way of providing safety services, it can be divided into periodic security message and emergency safety message. Periodic security message, also known as beacon frames, contains the basic state information of vehicle nodes. The another type of message is event-driven message, which mainly includes the early warnings of road natural disaster, front traffic accident, lane emergency change, road congestion, hidden obstacles, red light running, and cooperative driving anticollision. On the one hand, the lack of safety information may lead to the driver unable to avoid traffic accidents, overtaking, and other emergencies in time, which may lead to major traffic accidents. On the other hand, it may also make the driver unable to choose a reasonable route and enter a more crowded road section, aggravate the road congestion, and result in the dual waste of travel time and energy, which brings huge economic losses and terrible travel experience. Therefore, the dissemination of safety information is extremely important in the application of VANET for traffic safety. In the face of the ever-changing traffic road environment, how to carry out the high reliability of safety information transmission is the focus of this paper. 
Security messages usually need to reach a specific area quickly and efficiently to inform the area vehicles of some special or dangerous situations, so security applications are usually implemented by broadcast. However, the traditional broadcast algorithm is mainly pure flooding, where any node who receives the messages will forward it immediately. Although this algorithm can make every effort to ensure that messages cover the whole network, there are still some problems such as reliability of the messages and broadcast storm.

The reliability of the messages cannot be guaranteed. In the VANET network, the reliable transmission of security messages is very important to reduce vehicle traffic accidents. Reliability of traditional broadcast networks is achieved mainly through three measures: repeated flooding broadcasts, selection of reliable forwarding nodes (as shown in Figure 1), and cluster structures [2]. These three measures can improve the reliability to a certain extent but cannot explicitly determine whether the sending node successfully broadcasts information to neighbors. Based on this consideration, we introduce the error control technology to broadcast mechanism, which has been published in [3]. At the same time, the biggest contribution of this research is that the result can be compatible with all existing broadcast protocols, that is, adding feedback mechanism under the original protocol.

However, there are many problems in this mechanism. First, there is no communication between adjacent nodes who only communicate with their superiors in [3]. In the scene of dense vehicles, the vehicle network presents a grapelike cluster structure as shown in Figure 1. In this structure, there are more nodes in the communication range, as shown in Figure 1, some forwarding nodes are in adjacent positions, that is, within the communication range of each other. In this scenario, in addition to completing the transmission to the child node, the broadcast behavior of the node has a significant impact on the communication of the neighbor forwarding node. And this effect will increase with the vehicle density, so the communication between adjacent nodes cannot be ignored based on the broadcasting characteristics of radio waves [4]. And it will lead to the evolution of the tree-based broadcast network into the mesh broadcast network, as shown in Figure 2. So, how to solve this problem is our first consideration.

Second, the protocol proposed in [3] will further aggravate the broadcast storm problem. That is because the receiver also sends information in addition to the sender broadcasting. Therefore, under the proposed protocol, how to effectively reduce the broadcast storm is a problem that we cannot ignore.

Third, sending information of the receiver will further increase energy consumption. There are two roles in VANET, vehicle node and roadside system. With sufficient energy supply, broadcast energy saving is rarely considered. However, with the emergence of new energy vehicles, energy supply becomes limited. And how to improve the vehicle's endurance becomes crucial. At the same time, there will be frequent information interaction between communication nodes, especially the real-time broadcast behavior of security messages, which will seriously affect the endurance of new energy vehicles. Therefore, the design of energy-saving broadcast mechanism to realize the green communication of VANET is a problem.

In order to solve the above problem in this paper, considering the broadcast characteristics of wireless signal, we design and propose a broadcast scheme for VANET, which is of great significance to reduce traffic accidents and ensure the stable operation of the intelligent transportation system. The main contributions of this paper are as follows:

(1) Considering the broadcast characteristics of radio, the tree-based broadcast model is extended to a general mesh broadcast model, which is more suitable for the actual broadcast situation of the Internet of vehicles

(2) In the general mesh broadcast model, error control technology is used to ensure the reliable reception of the target

(3) The cooperative communication technology is introduced to analyze the mesh broadcast model, and the analytic expressions of broadcast delay, energy consumption, and other performance indicators are obtained, which provide theoretical support for broadcast communication

(4) The oiARQ and ieARQ technologies are introduced, to solve the broadcasting storm and energy consumption problems of the proposed protocol

\section{Related Work}

The pure flood broadcasting scheme is that each vehicle retransmits information after successfully receiving. However, the broadcast efficiency of the pure flood broadcasting scheme is very low. The main reason is poor scalability and information collision. As networks become more dense, the same information is broadcast repeatedly which results in redundancy and wasting the limited wireless channel bandwidth, so pure flooding cannot maintain scalability for network density. In addition, a large number of vehicles who simultaneously broadcast the same information can cause severe information collisions and also known as broadcast storm problems. Therefore, in order to solve these two problems, the typical way is to select some vehicles for forwarding, instead of repeating the broadcasting of all vehicles. Broadcast schemes are divided into five main categories according to the criteria for selecting forwarding nodes: geographic location-based [4-13], probability-based [14], [15], [16], [17], [18], [19], and [20], delay-based [21-25], clustering-based [26-28], and encoding based [29, 30]. In Dynamic Hybrid Broadcasting Protocol (DHBP) [9], to solve the broadcast storm problem, the relay node stops rebroadcasting depending on the value of decision-making function (DMF). And the DMF is related to packet level, distance, and data transmission times. In the reliable broadcast mechanism (RBM) [10], the reliability of the sender is determined by implicit acknowledgment. In short, the forwarding message of the next node is regarded as the 


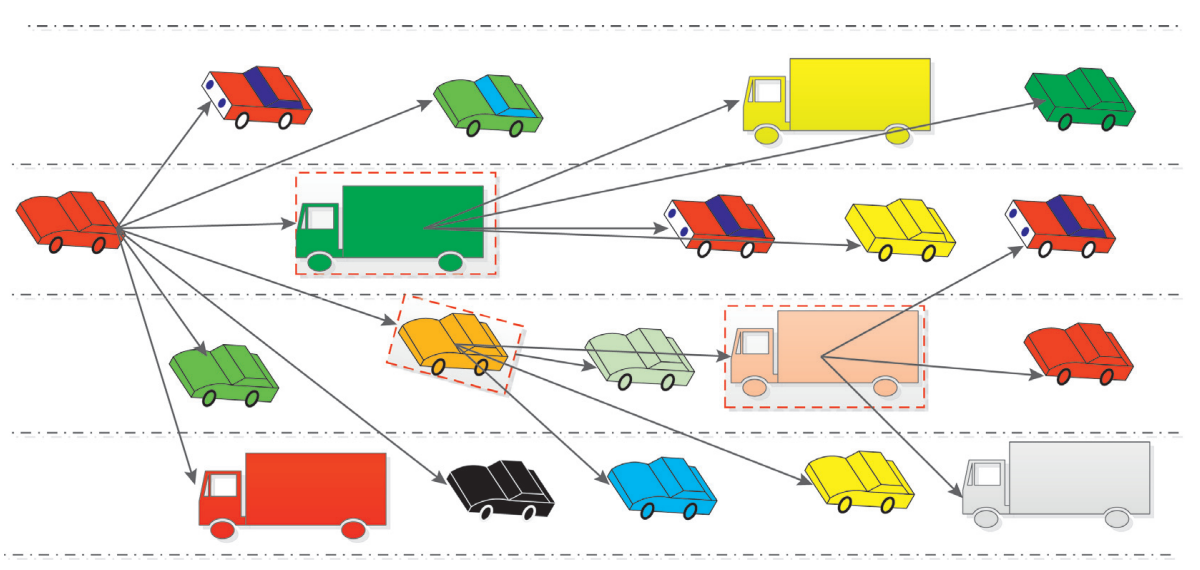

FIgURE 1: Grape-cluster-like characteristics of VANET.

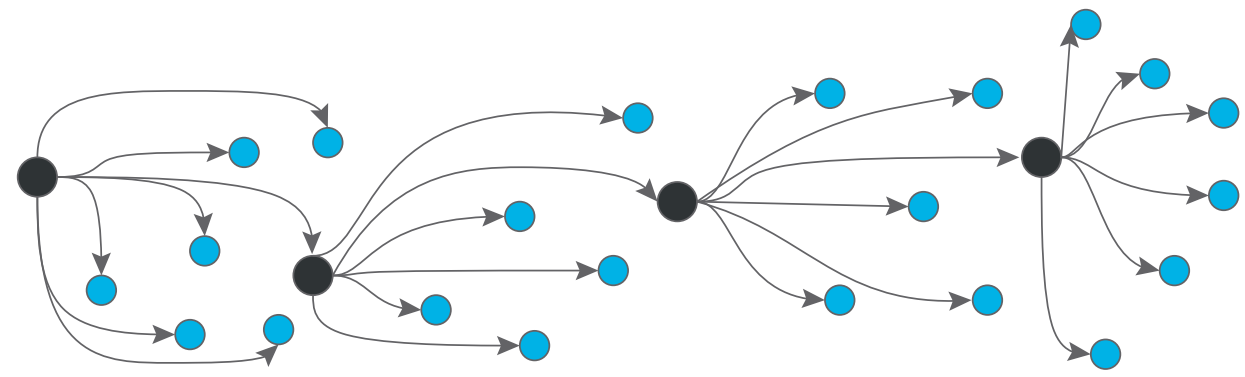

(a)

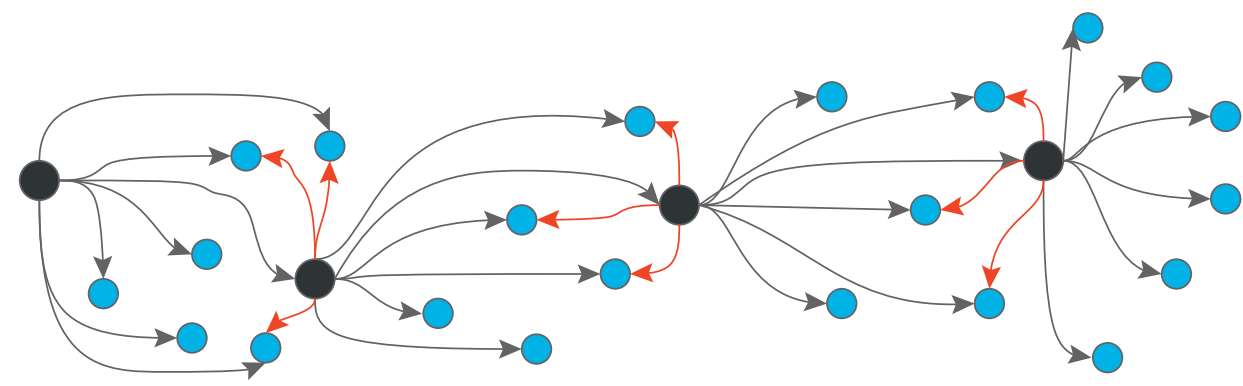

(b)

Figure 2: The influence of radio broadcast characteristics. (a) Tree-based broadcast architecture (ignoring the impact of forwarding nodes on historical nodes). (b) Mesh broadcast architecture (considering the impact of forwarding nodes on historical nodes).

successful transmission confirmation. At the same time, the system determines the priority of the forwarding node according to the predicted distance, density, and relative speed. In energy efficient-fast message distribution routing protocol (EE-MDRP) [11], the source vehicle found vehicle neighbors positions through using a vector-angle oriented classification model. Then, the optimal relay is the neighbor who responds to data with minimum message delivery time. In Opportunistic Directional-Location Added Routing (ODLAR) [12], the node with the highest link quality, the minimum angle deviation, and the minimum distance from the destination is selected as the relay node. The authors propose a position-based speed adaptive dissemination (PBSAD) approach in [13], where relay nodes for rebroadcast are selected considering the geographical position. And the time slots for rebroadcasting are related to the vehicle speed.
In the density-aware probabilistic interest forwarding (DAPIF) method [17], the forwarding probability depends on the neighbor density and the time distance from the sender. A fuzzy-based beaconless probabilistic broadcasting algorithm (FBBPA) is proposed in [19] to decide the packets forwarding probability of the relay node on the basis of their distance, angular orientation, movement direction, and buffer load delay. Volunteer's dilemma game for VANET broadcast (VDGVB) [25], the timer value of relay node, depends on previous transmission, forwarding probability and nearby density. In the Emergency Message Dissemination System (EMDS) [28], vehicles are grouped into clusters using their direction, speed, and relative location. Table 1 shows the comparison among the above discussed protocols.

However, when selecting forwarding nodes, these broadcast algorithms only use local information (such as distance, relative speed, vehicle location, and network 
TABLE 1: Comparison between the various broad protocols.

\begin{tabular}{|c|c|c|c|c|}
\hline Paper & Protocol & Problem & Forward strategy & Considerations \\
\hline [9] 2019 & DHPB & Broadcast storm & Location-based & Packet level, distance, and transmission times \\
\hline [17] 2020 & DAPIF & storm & ed & e distance \\
\hline [10] 2019 & $\mathrm{RBM}$ & Consumption, broadcast overhead & n-based & ative speed \\
\hline [11] 2020 & EE-FMDRP & educe delay, & & ice \\
\hline [12] 2020 & & orwarder & & $\mathrm{n}$, and distance \\
\hline [28] 2019 & c & Broadca & & Direction, speed, and relative location \\
\hline [25] 2019 & $\mathrm{~V}$ & Broadca & I & Transmission, foru \\
\hline [13] 2020 & & $\mathrm{Br}$ & $\mathrm{Lc}$ & based \\
\hline [19] 2020 & A & rerhead & sed & distance, orientation, direction, and buffer delay \\
\hline [20] 2019 & Protocol & Max network capacity & Probability-based & Optimal stopping theory \\
\hline
\end{tabular}

density). The transmission range of the forwarding node cannot cover the specified area, and the reliability problem of receiver is still not guaranteed.

So, in order to achieve reliable of receiver, we have already presented a version of our scheme in [3]. We introduced ARQ feedback mechanism for the first time in the broadcasting mechanism and proposed the SW-HBH-ARQ broadcasting scheme based on the tree-structure network. However, there are three problems in this mechanism.

First, there is no communication between adjacent nodes in [3]. Especially, in the scenario where the sender is one and the receivers are multiple, it is assumed that the receivers can only accept the data from the sender while ignoring the same data from the brothers. However, this assumption is not suitable for the actual communication scenario, where the communication between forwarding nodes is continuous based on the broadcasting characteristics of radio waves. Therefore, the mesh network is the most appropriate way to describe the Internet of vehicles communication. Then, how to extend the conclusion to the mesh network is a difficulty.

As shown in Figure 3, based on the characteristics of radio waves, when the receiver successfully receives the data and forwards the data, the failed brothers can also receive the data. This method can not only effectively improve the broadcast success rate but also expand the tree network to the general mesh network through the link of the brothers, as shown in Figure 4. The above transmission principle is also known as collaborative communication technology. Therefore, we introduced the collaborative communication technology into the data broadcasting mechanism which extended the tree-based network to the general mesh network for the first time.

Second, the protocol with feedback mechanism will aggravate the broadcast storm problem, because the receiving node is also sending information. Table 1 shows the related research studies in recent years. It can be seen from the list that most studies solve the broadcast storm by selecting the optimal forwarding node or cluster to ensure the efficient distribution of information. Obviously, these results are what we need, and the feedback-based protocol can be compatible with these broadcast strategies. However, there is still no reduction in the number of feedback at the receiving end. Therefore, how to reduce the number of

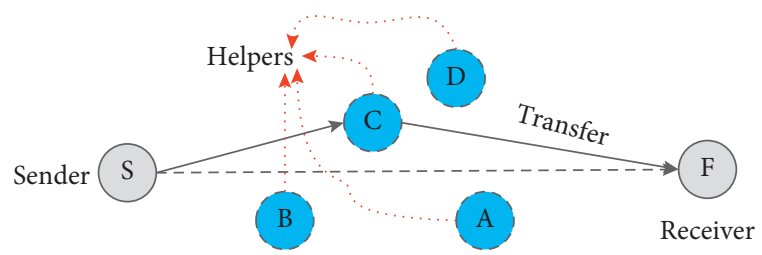

FIgURE 3: Collaborative communication technology.

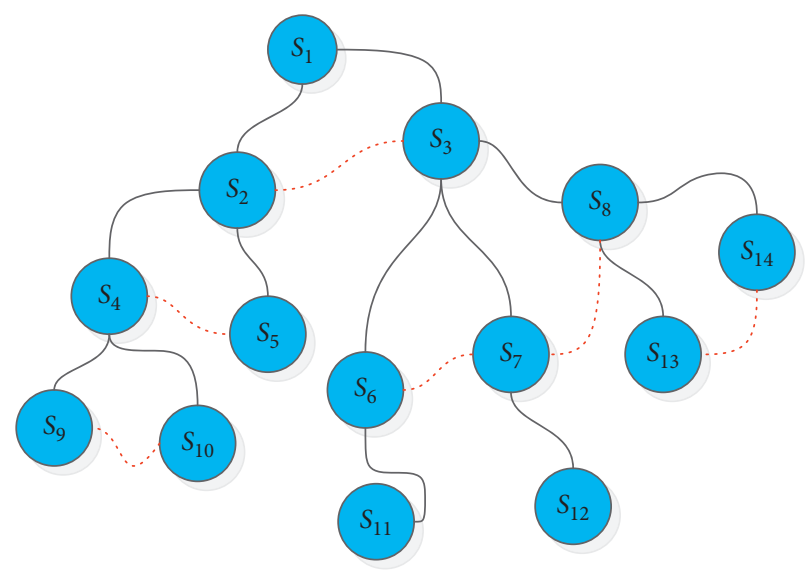

Figure 4: Mesh network structure.

feedback is the key problem to solve the broadcast storm in our proposed protocol.

Third, the feedback from the receiver will also increase the energy consumption of the system. This problem can be solved together with problem two. Obviously, reducing the number of feedback can also achieve energy saving.

In this paper, under the premise of ensuring the reliability of the receivers, the tree-based network is extended to the general mesh network by introducing cooperative communication technology. In order to minimize the system energy consumption and broadcast storm, four data cooperative broadcasting mechanisms are proposed in the mesh network. Finally, the quantitative analysis of system energy consumption and delay under the four mechanisms is carried out to provide theoretical support for the planning of the mesh broadcast network. 


\section{Cooperative Broadcasting Scheme Based on Mesh Network Structure}

In this section, based on the mesh network structure, four cooperative broadcasting schemes SW-HBH-CARQ, SWHBH-oiCARQ, SW-HBH-ieCARQ, and SW-HBH-oieCARQ are proposed, and then the performance such as energy consumption and delay are analyzed separately. For analysis purposes, the symbols used in this paper and their descriptions are given in Table 2 .

3.1. SW-HBH-CARQ Broadcast Scheme and Analysis. In [31], the SW-HBH-ARQ protocol is mentioned for the first time, and its energy consumption is analyzed briefly. This section supplements the process of the protocol and discusses the delay.

As shown in Figure 5, the retransmission mechanism of SW-HBH-CARQ broadcast protocol is established on each hop. At any hop, the parent stops retransmission until it receives the ACK from all children. Different from ARQ protocol, the child will return ACK in two cases. One is that the child successfully receives the data from the parent (the same as ARQ protocol), and the other is that the subterminal does not receive the data from the parent and will wait for the cooperation of the siblings. When it successfully receives the data from the siblings, it will also return ACK to the parent. The time from the parent broadcasting data to the subterminal returning feedback is recorded as data transmission cycle. After a data transmission cycle, the parent judges the ACK number of receiving. If the number is the same as that of the children, the parent starts to broadcast the next data. Otherwise, the parent retransmits the current data. The specific implementation steps are shown in Table 3.

This step is applicable to each hop. First, the prebroadcast packets in the buffer of the parent are numbered $j(j=1,2,3, \ldots)$, and the number of its subterminals is recorded as $N_{i} ; i$ is initialized as 0 .

For the parent, cooperative communication technology increases the probability of broadcasting and reduces the probability of retransmission, thus reduces the energy consumption of the system. For subterminals, cooperative communication technology increases the probability of acception and increases the probability of feedback, so as to increase the feedback energy consumption. Therefore, the impact of SW-HBH-CARQ broadcast protocol on system energy consumption is determined by its reliability, feedback and broadcast probability, and other parameters.

In [31], we have discussed how to set the maximum retransmission number of any parent on the premise of ensuring the reliability. Note that the system reliability is $\beta$, that is, the reliability of the link $s_{I_{0}}, s_{I_{1}}, s_{I_{2}}, \ldots, s_{I_{M_{I}-1}}, s_{I}$ with the lowest transmission success rate is $\beta$, the number of hops of the link is $M_{I}$, and the system reliability is evenly allocated
TABle 2: Symbols and descriptions.

\begin{tabular}{lc}
\hline Sym & Descriptions \\
\hline$W$ & The network \\
$E$ & Energy-consuming \\
$s_{1}$ & Root \\
$s_{i}$ & ID of node \\
$s_{b(i)}$ & The sibling of $s_{i}$ \\
$s_{p(i)}$ & The parent of $s_{i}$ \\
$\tau_{b}$ & The broadcast delay \\
$\tau_{f}$ & The feedback delay \\
$f_{i}$ & Feedback rate of $s_{i}$ \\
$I_{j}$ & Link from root to $s_{j}$ \\
$p_{i}$ & Broadcast rate of $s_{i}$ \\
$p_{c(i)}$ & Broadcast rate of $s_{c(i)}$ \\
$p_{b(i)}$ & Broadcast rate of $s_{b(i)}$ \\
$p_{p(i)}$ & Broadcast rate of $s_{p(i)}$ \\
$s_{c(i)}$ & The child/subterminal of $s_{i}$ \\
$M_{i}$ & Minimal hop from $s_{i}$ to $s_{1}$ \\
$q_{i}$ & Feedback rate from child to $s_{i}$ \\
$N_{i}$ & The children number of $s_{i}$ \\
$M_{I}$ & The hops number of link $I$ \\
$I$ & Minimum reliability line link \\
$\beta$ & System reliability, \\
$Y_{i}$ & Feedback energy of $s_{i}$ \\
$R$ & The number of terminals \\
$X_{i}$ & The broadcast energy of $s_{i}$ \\
$N_{c(i)}$ & Grandchildren number of $s_{i}$ \\
$\tau_{s}$ & The listening delay \\
$T_{X}$ & Packet delay of X protocol \\
$L_{X}$ & Broadcasting rate of $s_{i}$ \\
$a_{i}$ & Transmission cycle of $s_{i}$ \\
$v(i)$ &
\end{tabular}

to each hop under SW-HBH-CARQ protocol; that is, for any hop, the reliability is $\beta^{\left(1 / M_{I}-1\right)}$.

Then, the minimum value $L_{\mathrm{CARQ}}\left(M_{i}, \beta\right)=(\log$ $\left.\left(1-\beta^{\left(1 / M_{I}-1\right)}\right) / \log \left(1-p_{I_{i}}\right)\right)$ is taken as the maximum retransmission number of $s_{i}$, where $M_{i}$ is the hop number from $s_{i}$ to root.

Here, we find the minimum value of the maximum retransmissions number to meet the system reliability and get the minimum energy consumption, so as to effectively extend the system life. At the same time, compared with the CARQ protocol and ARQ protocol, the maximum retransmission number is equal under the same system reliability. This is because the system reliability used in this paper is only related to the link with lowest transmission rate and has nothing to do with whether the siblings participate in the cooperation. Thus, in the next three proposed schemes (SW-HBH-oiCARQ, SW-HBHieCARQ, and SW-HBH-oieCARQ), the maximum number of retransmissions is no longer calculated, and this conclusion is directly used.

In [31], the energy consumption of the system under $\mathrm{SW}-\mathrm{HBH}-\mathrm{CARQ}$ protocol is also obtained as follows: 


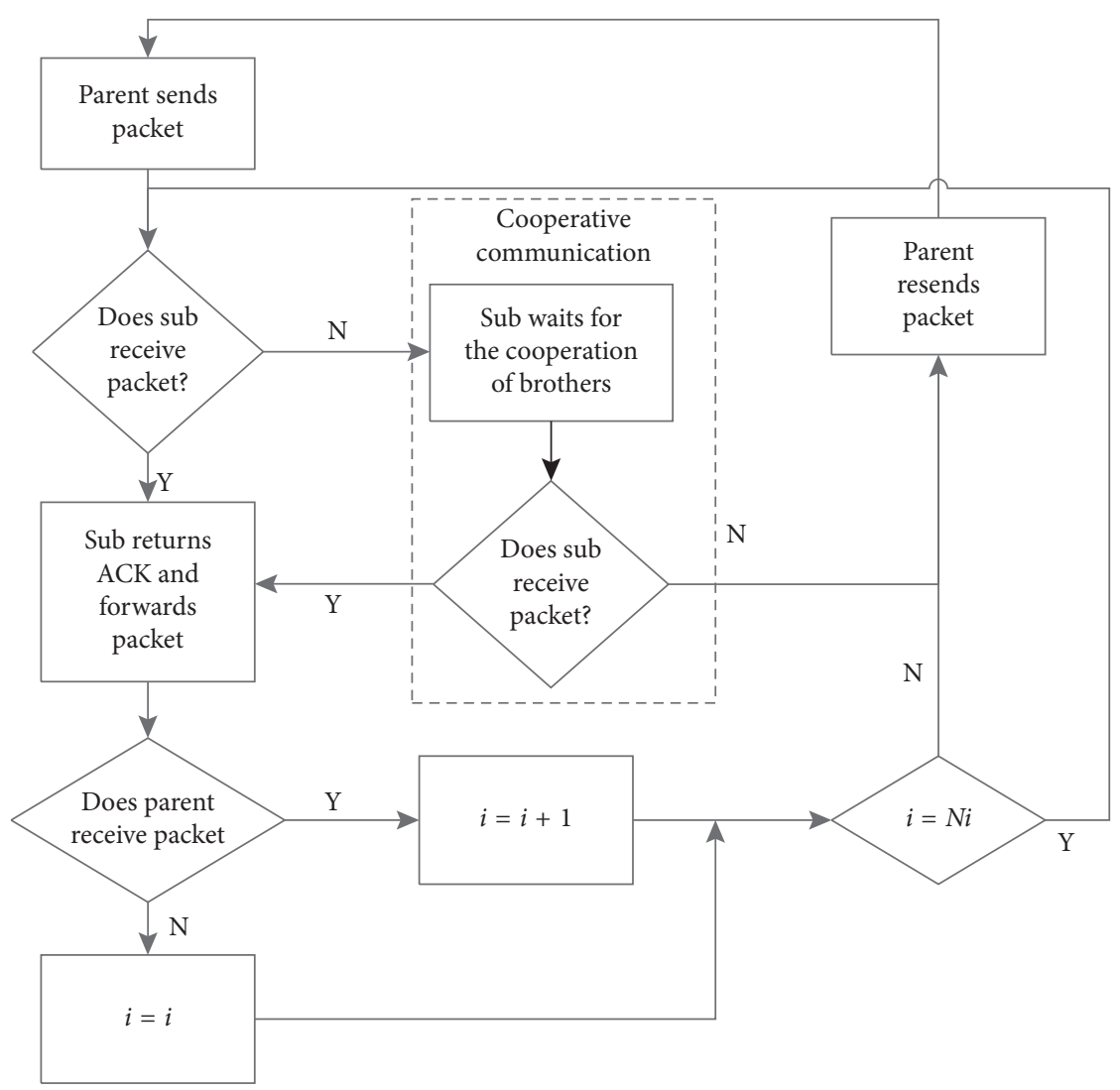

Figure 5: Flowchart of SW-BHB-CARQ protocol.

TABLE 3: Implementation steps of SW-HBH-CARQ protocol.

Step 1: the parent starts to broadcast the packet (ID. $j$ ) and initializes $i$ as 0 .

Step 2: the subterminal judges whether the packet is successfully received. If so, it advances to step 3; otherwise, it advances to step 4. Step 3: the subterminal forwards the data and returns the ACK to parent; then, it advances to step 5.

Step 4: the subterminal waits for the cooperation data from siblings. If the subterminal receives correct data, it returns to step 3; otherwise, it advances to step 5 .

Step 5: the parent judges whether it receives the ACK, if so, $i=i+1$; otherwise, $i=i$.

Step 6: after a data transmission cycle, if $i=N_{i}$, then $j=j+1$, if $i<N_{i}$, then $j=j$, and it returns to step 1 .

$$
E(W)=\sum_{i=1}^{R}\left(E\left(X_{i}\right)+f_{i} E\left(X_{p(i)}\right)\right)
$$

where $E\left(X_{i}\right)=\left(1-\left(1-a_{i}\right)^{L_{C A R Q}\left(M_{I}, \beta\right)} / a_{i}\right)$ is the broadcast energy of $s_{i}$,

$$
a_{i}=\left(q_{i}\right)^{N_{i}} \sum_{k=1}^{N_{i}} C_{N_{1}}^{k}\left(p_{i}\right)^{k}\left(1-p_{i}\right)^{N_{i}-k} \overbrace{\left(1-\left(1-p_{c(1)}\right)^{k}\right) \cdot \ldots \cdot\left(1-\left(1-p_{c(1)}\right)^{k}\right)}^{N_{i}-k},
$$

is the broadcasting probability of $s_{i}$, and

$$
f_{i}=p_{p(i)}+\left(1-p_{p(i)}\right) \sum_{k=1}^{N_{1}-1} C_{N_{i}}^{k}\left(1-p_{p(i)}\right)^{N_{i}-k}\left(p_{p(i)}\right)^{k}\left(1-\left(1-p_{b(i)}\right)^{k}\right),
$$


is the feedback probability. $E\left(Y_{i}\right)=f_{i} E\left(X_{p(i)}\right)$ is feedback energy consumption of $s_{i}$.

In particular, assuming that all the siblings have the same transmission probability, the feedback energy of any terminal is only related to the parent and the siblings, in which the feedback energy consumption of each subterminal is the same. Thus, considering that $s_{i}$ has $N_{i}$ children, according to the above formula, $E\left(\Delta_{i}\right)$ is the sum of the feedback energy of all children of $s_{i}$, and then $E\left(\Delta_{i}\right)=N_{i} f_{i} E\left(X_{i}\right)$. So, the total energy consumption is

$$
E_{\mathrm{CARQ}}=\sum_{i=1}^{R}\left(E\left(X_{i}\right)+E\left(\Delta_{i}\right)\right)=\sum_{i=1}^{R}\left(1+N_{i} f_{i}\right) E\left(X_{i}\right) \text {. }
$$

Finally, the broadcast delay is calculated. Obviously, the delay of each hop is related to the retransmissions number. The broadcast delay is $\tau_{b}$, and the feedback delay is $\tau_{f}$. Considering that the subterminals participate in cooperative communication, then the data transmission period is $2 \tau_{b}+\tau_{f}$, and the delay of SW-HBH-CARQ broadcast protocol is obtained as follows:

$$
T_{\text {CARQ }}=\sum_{j=1}^{M} \max _{\substack{1<i \leq R \\ M_{i}=j}}\left(E\left(X_{i}\right)\left(2 \tau_{b}+\tau_{f}\right)\right)
$$

where $M$ is the maximum hops number and $M_{i}$ is the hops number from $s_{i}$ to $s_{1}$.

\subsection{SW-HBH-oiCARQ Broadcast Scheme and Analysis.} In the SW-HBH-oiCARQ protocol, using the broadcast characteristics of the subterminal, the rebroadcast message of the child forwarder is treated as implicit acknowledgment (iACK). If the overhearing is successful, it is considered that the subterminal successfully feeds back iACK. Therefore, all intermediate terminals need not report back eACKs (explicit ACKs) directly, which is more energy-saving than SW$\mathrm{HBH}-\mathrm{CARQ}$ protocol [10].
As shown in Figure 6, after the parent broadcasting, if the subterminal receives the data, then it forwards them. This forwarding has three purposes: one is to forward the data to its next terminals, the other is as feedback to the parent, and the third is to forward the data to its sibling as a collaborator. When the subterminal fails to receive the data, it firstly waits for the cooperation from siblings. After the successful cooperation, the subterminal forwards the data meanwhile as an iACK for parent. Otherwise, the parent retransmits the data. The parent will start to broadcast the next data when it had received the iACKs from all children. Especially, the subterminal feeds back the eACK instead iACK if it is a leaf terminal. The specific implementation steps are shown in Table 4. These steps are suitable for each hop.

In the SW-HBH-oiCARQ broadcast protocol, only the leaf terminal returns the eACK, and all the intermediate terminals do not send feedback. Therefore, compared with the SW-HBH-CARQ broadcast protocol, the SW-HBHoiCARQ broadcast protocol is more energy efficient. In this section, we only consider the overhearing delay of the parent and ignore the overhearing energy.

In this section, we first discuss the energy consumption of SW-HBH-oiCARQ broadcast protocol. We use the assumption in [3] that the feedback probability relationship between iACK and eACK is $r_{i} q_{i}$. Then, for the source $s_{1}$, there are $k\left(1 \leq k \leq N_{1}\right)$ subterminals received the data $C_{N_{1}}^{k} p_{1}^{k}\left(1-p_{1}\right)^{N_{1}-k}$. If the failed $N_{1}-k$ subterminals can successfully receive the forwarding data from the siblings, now all subterminals have received the data and then return the feedback. After all feedback is reached to $s_{1}, s_{1}$ stops retransmission. The probability can be expressed as follows:

$$
\left(r_{1} q_{1}\right)^{N_{1}} \overbrace{\left(1-\left(1-p_{c(1)}\right)^{k}\right) \ldots\left(1-\left(1-p_{c(1)}\right)^{k}\right)}^{N_{1}-k} .
$$

If $a_{1}^{o i}$ is the probability of $s_{1}$ stopping retransmission under SW-HBH-oiCARQ protocol,

$$
a_{1}^{o i}=\left(r_{1} q_{1}\right)^{N_{1}} \sum_{k=1}^{N_{1}} C_{N_{1}}^{k}\left(p_{1}\right)^{k}\left(1-p_{1}\right)^{N_{1}-k} \overbrace{\left(1-\left(1-p_{c(1)}\right)^{k}\right) \cdot \ldots \cdot\left(1-\left(1-p_{c(1)}\right)^{k}\right)}^{N_{1}-k} .
$$

Then, the average broadcast energy consumption of $s_{1}$ is

$$
E^{o i}\left(X_{1}\right)=L_{\mathrm{CARQ}}\left(M_{1}, \beta\right)\left(1-a_{1}^{o i}\right)^{L_{\mathrm{CARQ}}\left(M_{1}, \beta\right)-1}+\sum_{k=1}^{L_{\mathrm{CARQ}}\left(M_{1}, \beta\right)-1} k\left(1-a_{1}^{o i}\right)^{k-1} a_{1}^{o i}=\frac{1-\left(1-a_{1}^{o i}\right)^{L_{\mathrm{CARQ}}\left(M_{1}, \beta\right)}}{a_{1}^{o i}} .
$$




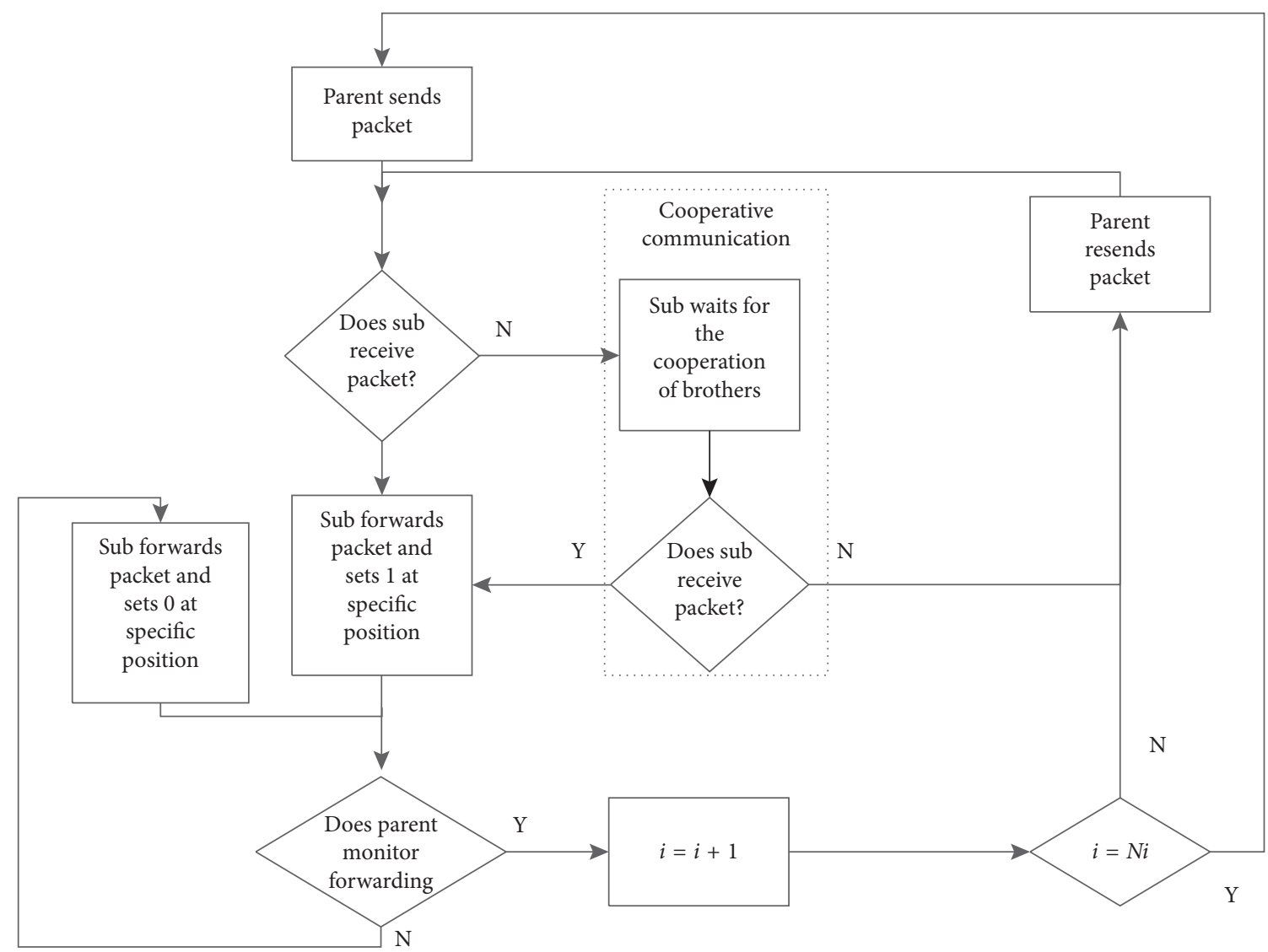

FIgURE 6: Flowchart of SW-BHB-oiCARQ protocol.

TABLE 4: Implementation steps of the SW-HBH-oiCARQ protocol.

Step 1: the parent broadcasts data packet (ID. $j$ ) and initializes $i$ as 0 .

Step 2: the subterminal judges whether the packet is successfully received. If so, it advances to step 3; otherwise, it advances to step 5. Step 3: the subterminal determines whether it is a leaf terminal. If so, it directly returns eACK to the parent. Otherwise, it advances to step 4. Step 4: the subterminal determines whether it receives the packet for the first time. If so, it will set the special position as 1 , otherwise as 0 , and forwards the data, then advances to step 6.

Step 5: the subterminal waits for the cooperation data from siblings. If it successfully receives the data, it returns to step 3; otherwise, it advances to step 6.

Step 6: the parent judges whether it overhears the forwarding or receives the eACK from child. If so, then $i=i+1$; otherwise, $i=i$. Step 7: after a transmission cycle, if $i=N_{i}$, then $j=j+1$, if $i<N_{i}$, then $j=j$, and then it return to step 1 .

Similarly, for any terminal $s_{i}$, its broadcast energy is

$$
E^{o i}\left(X_{i}\right)=\frac{1-\left(1-a_{i}^{o i}\right)^{L_{\mathrm{CARQ}}\left(M_{I}, \beta\right)}}{a_{i}^{o i}}
$$

where $N_{i}$ is the subterminal number of $s_{i}$, and the broadcasting probability of $s_{i}$ is

$$
a_{i}^{o i}=\left(r_{i} q_{i}\right)^{N_{i}} \sum_{k=1}^{N_{i}} C_{N_{i}}^{k}\left(p_{i}\right)^{k}\left(1-p_{i}\right)^{N_{i}-k} \overbrace{\left(1-\left(1-p_{c(i)}\right)^{k}\right) \cdot \ldots \cdot\left(1-\left(1-p_{c(i)}\right)^{k}\right)}^{N_{i}-k} .
$$

Next, the feedback energy of the system is calculated. In SW-HBH-oiCARQ protocol, all intermediate terminals (non-leaf terminals) have no feedback, and only leaf terminals will return eACKs. For any leaf terminal $s_{i}$ whose brothers do not forward data, there are no cooperative communications. So, we only calculate feedback energy of $s_{i}$ if it receives data from the parent, and the probability is $p_{p(i)}$. If $f_{i}^{o i}$ is the feedback probability of $s_{i}$, 


$$
f_{i}^{o i}=\left(\begin{array}{ll}
0, & N_{i}>0, \\
p_{p(i)}, & N_{i}=0 .
\end{array}\right.
$$

Then, the feedback energy of $s_{i}$ is expressed as $E^{o i}\left(Y_{i}\right)=f_{i} E^{o i}\left(X_{p(i)}\right)$. And the total energy consumption of the system can be further obtained as follows:

$$
E^{o i}(W)=\sum_{i=1}^{R}\left(E^{o i}\left(X_{i}\right)+f_{i}^{o i} E^{o i}\left(X_{p(i)}\right)\right) .
$$

In particular, all siblings have the same transmission probabilities, i.e.,

$$
E^{o i}\left(\Delta_{i}\right)=N_{i} f_{i}^{o i} E^{o i}\left(X_{i}\right) .
$$

At this time, the system energy consumption is

$$
E_{\mathrm{CARQ}}^{o i}=\sum_{i=1}^{R}\left(E^{o i}\left(X_{i}\right)+E^{o i}\left(\Delta_{i}\right)\right)=\sum_{i=1}^{R}\left(1+N_{i} f_{i}^{o i}\right) E^{o i}\left(X_{i}\right) .
$$

Next, the system delay is calculated. The transmission delay of each hop is related to the retransmissions number. The overhearing delay is recorded as $\tau_{s}\left(\tau_{s}>\tau_{f}\right)$. And the transmission cycle of $s_{i}$ is

$$
v(i)= \begin{cases}2 \tau_{b}+\tau_{s}, & N_{c(i)}>0, \\ 2 \tau_{b}+\tau_{f}, & N_{c(i)}=0,\end{cases}
$$

where $N_{c(i)}$ is the number of grandchildren of $s_{i}$.

Then, the delay of SW-HBH-oiCARQ broadcast protocol is

$$
T_{\text {CARQ }}^{o i}=\sum_{j=1}^{M} \max _{\substack{1<i \leq R \\ M_{i}=j}} E\left(X_{i}\right) v(i) .
$$

3.3. SW-HBH-ieCARQ Broadcast Scheme and Analysis. In SW-HBH-ieCARQ broadcast protocol, as shown in Figure 7 , for all children, they forward the data after received data for the first time, and then the parent overhears the forwarding as iACKs. Especially, they forward the data and directly return the eACKs after receiving the data again.

In SW-HBH-ieCARQ broadcast protocol, for any intermediate terminal, it forwards the data when it successfully receives the data (from the parent or brothers) for the first time; meanwhile, the parent overhears the forwarding as iACK. The overhearing can reduce the feedback energy but greatly increases the length of transmission cycle. Therefore, in the next section, we introduce SW-HBH-oieCARQ protocol in which the terminal who received data from siblings will directly return eACK instead iACK. By increasing the feedback energy, the length of transmission cycle is reduced. And these two protocols are compared in the numerical analysis section.

The specific implementation steps of the SW-HBHieCARQ broadcast protocol are shown in Table 5.
From Figure 7, in SW-HBH-ieCARQ protocol, it can be seen that for any terminal, it only sends one eACK less than $\mathrm{SW}-\mathrm{HBH}-\mathrm{CARQ}$ protocol. Then, the system energy is

$$
E_{\mathrm{CARQ}}^{i e}=E_{\mathrm{CARQ}}-(R-1)=\sum_{i=1}^{R}\left(1+N_{i} f_{i}\right) E\left(X_{i}\right)-(R-1) .
$$

Next, the delay is calculated. Considering that the subterminal participates in cooperative communication and the parent overhears the forwarding, the transmission cycle $v(i)$ of $s_{i}$ who receives the data for the first time is

$$
v(i)= \begin{cases}2 \tau_{b}+\tau_{s}, & N_{c(i)}>0, \\ 2 \tau_{b}+\tau_{f}, & N_{c(i)}=0 .\end{cases}
$$

According to the SW-HBH-ieCARQ broadcast protocol, when $s_{i}$ receives the same data again, it directly returns eACK. So, the transmission cycle is $2 \tau_{b}+\tau_{f}$, and the delay of the SW-HBH-ieCARQ broadcast protocol is

$$
T_{\text {CARQ }}^{i e}=\sum_{j=1}^{M} \max _{\substack{1<i \leq R \\ M_{i}=j}}\left[\left(E\left(X_{i}\right)-1\right)\left(2 \tau_{b}+\tau_{f}\right)+v(i)\right] .
$$

3.4. SW-HBH-oieCARQ Broadcast Scheme and Analysis. In the SW-HBH-iARQ protocol, after the parent broadcasts data, this paper considers the following situations. The subterminal failed to accept data from the parent but received data from siblings, and then it forwards data also as iACK. It is worth mentioning that failed broadcasting indicates the harsh channel condition, which also means that the parent has a quite lower overhearing probability. Considering the relationship $r_{i} q_{i}<q_{i}$, which means the acceptance probability of eACK is greater than the overhearing probability, so the SW-HBH-oieARQ protocol is introduced to increase the feedback reliability.

As shown in Figure 8, if the child receives data from the parent, it feeds back iACK according to oiARQ protocol; if it receives data from the siblings, it feeds back eACK directly. The specific implementation steps are shown in Table 6.

Based on the SW-HBH-oiCARQ protocol, the feedback mechanism of part subterminals is changed and further affects the retransmission energy.

First, the broadcast energy of SW-HBH-oieCARQ protocol is discussed as follows.

For source terminal $s_{1}$, if there are $k\left(1 \leq k \leq N_{1}\right)$ subterminals accepted data $C_{N_{1}}^{k} p_{1}^{k}\left(1-p_{1}\right)^{N_{1}-k}$, the failed $N_{1}-$ $k$ children receive forwarding data from siblings. Now, all subterminals successfully receive data. After $k$ subterminals forwarding are overheard and $N_{1}-k$ subterminals return eACK, then $s_{1}$ stops retransmission, and the probability can be expressed as

$$
\left(q_{1}\right)^{N_{1}-k}\left(r_{1} q_{1}\right)^{k} \overbrace{\left(1-\left(1-p_{c(1)}\right)^{k}\right) \ldots\left(1-\left(1-p_{c(1)}\right)^{k}\right)}^{N_{1}-k} .
$$




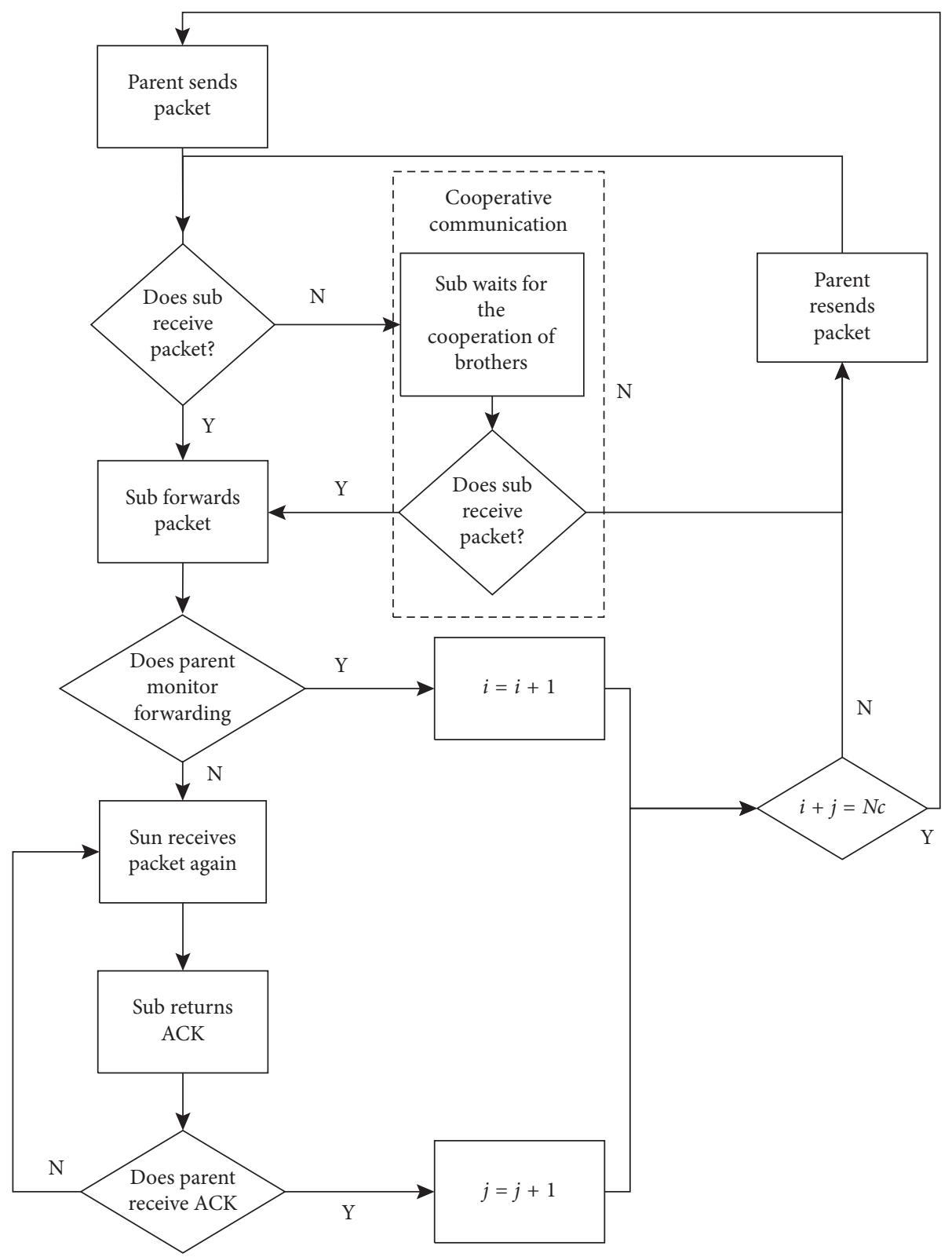

Figure 7: Flowchart of SW-BHB-ieCARQ protocol.

TABLE 5: Implementation step of SW-HBH-ieCARQ protocol.

Step 1: the parent starts broadcasting the packet (ID. $j$ ) and initializes $i$ as 0 .

Step 2: the subterminal determines whether the packet was successfully received, and if so, it advances to step 3; otherwise, it advances to step 5 .

Step 3: the subterminal determines whether it is a leaf terminal. If so, it returns ACK directly to the parent; otherwise, it advances to step 4. Step 4: the subterminal determines whether it receives the packet for the first time. If so, it forwards the data; otherwise, it returns the ACK directly and advances to step 6 .

Step 5: the subterminal waits for the cooperation data from siblings. And it returns to step 3 if it receives the correct data; otherwise, it advances to step 6.

Step 6: the parent determines whether it overhears the forwarding or receives ACK from the child, if so, $i=i+1$; otherwise, $i=i$. Step 7: after a transmission cycle, if $i=N_{i}$, then $j=j+1$, if $i<N_{i}$, then $j=j$, and return to step 1 . 
If $a_{1}^{\text {oie }}$ is remembered as the stopping retransmission probability of $s_{1}$,

$$
a_{1}^{\text {oie }}=\sum_{k=1}^{N_{1}}\left(q_{1}\right)^{N_{1}-k}\left(r_{1} q_{1}\right)^{k} C_{N_{1}}^{k}\left(p_{1}\right)^{k}\left(1-p_{1}\right)^{N_{1}-k} \overbrace{\left(1-\left(1-p_{c(1)}\right)^{k}\right) \cdot \ldots \cdot\left(1-\left(1-p_{c(1)}\right)^{k}\right)}^{N_{1}-k} .
$$

Then, the average broadcast energy of $s_{1}$ under SW-

$\mathrm{HBH}$-oieCARQ protocol is

$$
E^{o i e}\left(X_{1}\right)=L_{\mathrm{CARQ}}\left(M_{1}, \beta\right)\left(1-a_{1}^{o i e}\right)^{L_{\mathrm{CARQ}}\left(M_{1}, \beta\right)-1}+\sum_{k=1}^{L_{\mathrm{CARQ}}\left(M_{1}, \beta\right)-1} k\left(1-a_{1}^{o i e}\right)^{k-1} a_{1}^{\text {oie }}=\frac{1-\left(1-a_{1}^{o i e}\right)^{L_{\mathrm{CARQ}}\left(M_{1}, \beta\right)}}{a_{1}^{o i e}} .
$$

Similarly, for any terminal $s_{i}$ and $E^{\text {oie }}\left(X_{i}\right)=\left(1-\left(1-a_{i}^{\text {oie }}\right)^{L_{\mathrm{CARQ}}\left(M_{I}, \beta\right)} / a_{i}^{\text {oie }}\right)$, where $N_{i}$ is the subterminal number of $s_{i}$, the broadcast probability of $s_{i}$ is

$a_{i}^{\text {oie }}=\sum_{k=1}^{N_{i}}\left(q_{1}\right)^{N_{1}-k}\left(r_{1} q_{1}\right)^{k} C_{N_{1}}^{k}\left(p_{i}\right)^{k}\left(1-p_{i}\right)^{N_{i}-k} \overbrace{\left(1-\left(1-p_{c(i)}\right)^{k}\right) \cdot \ldots \cdot\left(1-\left(1-p_{c(i)}\right)^{k}\right)}^{N_{i}-k}$.

Next, the feedback energy of SW-HBH-oieCARQ protocol is discussed. For any terminal $s_{i}$, its feedback energy is 0 when accepting data from the parent and its feedback energy is 1 when accepting data from the siblings. And the analysis is as follows.

When $s_{i}$ accepts the wrong data, there are $k$ siblings received the data $C_{N_{i}}^{k}\left(1-p_{p(i)}\right)^{N_{i}-k}\left(p_{p(i)}\right)^{k}$. After $s_{i}$ receives the forwarding data from the siblings $1-\left(1-p_{b(i)}\right)^{k}$, then its feedback energy consumption is 1 .

Under the SW-HBH-oieCARQ protocol, if $f_{i}^{\text {oie }}$ is the feedback probability of $s_{i}$, then according to the full probability formula,

$$
f_{i}^{o i e}=\left(1-p_{p(i)}\right) \sum_{k=1}^{N_{1}-1} C_{N_{i}}^{k}\left(1-p_{p(i)}\right)^{N_{i}-k}\left(p_{p(i)}\right)^{k}\left(1-\left(1-p_{b(i)}\right)^{k}\right) .
$$

Then, the feedback energy of $s_{i}$ is expressed as $E^{\text {oie }}\left(Y_{i}\right)=f_{i}^{\text {oie }} E^{\text {oie }}\left(X_{p(i)}\right)$. The total energy consumption is obtained as follows:

$$
E^{\text {oie }}(W)=\sum_{i=1}^{R}\left(E^{o i e}\left(X_{i}\right)+f_{i}^{o i e} E^{o i e}\left(X_{p(i)}\right)\right) .
$$

Especially, if all siblings have the same transmission probability,

$$
E^{\text {oie }}\left(\Delta_{i}\right)=N_{i} f_{i} E^{\text {oie }}\left(X_{i}\right),
$$

while the total energy consumption of the system is as follows:

$$
E_{\mathrm{CARQ}}^{o i e}=\sum_{i=1}^{R}\left(E^{\text {oie }}\left(X_{i}\right)+E^{o i e}\left(\Delta_{i}\right)\right)=\sum_{i=1}^{R}\left(1+N_{i} f_{i}^{o i e}\right) E^{o i e}\left(X_{i}\right) .
$$

Next, the system delay is started calculating. It is remembered that the broadcasting delay is $\tau_{b}$, the feedback delay is $\tau_{f}$, and the overhearing delay is $\tau_{s}\left(\tau_{s}>\tau_{f}\right)$. Then, the transmission period in the network is

$$
v(i)= \begin{cases}2 \tau_{b}+\tau_{s}, & N_{c(i)}>0, \\ 2 \tau_{b}+\tau_{f}, & N_{c(i)}=0 .\end{cases}
$$

Then, the delay of SW-HBH-oieCARQ broadcast protocol is 


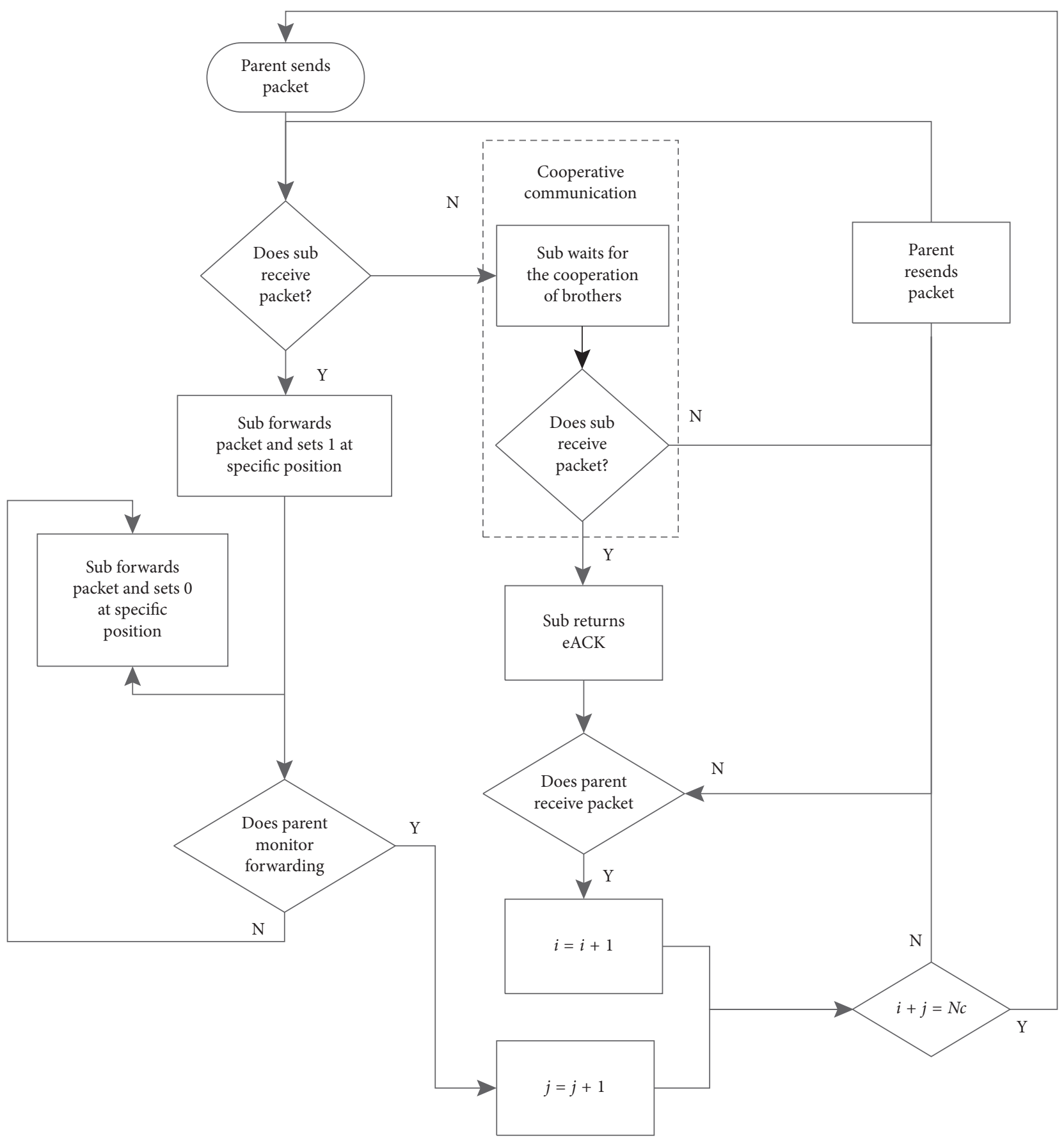

FIGURE 8: Flowchart of SW-BHB-oieCARQ protocol.

TABLE 6: Implementation step of SW-HBH-oieCARQ protocol.

Step 1: the parent starts broadcasting the packet (ID. $j$ ) and initializes $i$ as 0 .

Step 2: the subterminal determines whether the packet was successfully received, and if so, it advances to step 3; otherwise, it advances to step 5.

Step 3: the subterminal determines whether it is a leaf terminal. If so, it returns ACK directly to the parent; otherwise, it advances to step 4. Step 4: the subterminal determines whether it receives the data for the first time. If so, it will forward the data with a special location of 1. Otherwise, it will forward the data to step 6 with a special location of 0 .

Step 5: the subterminal waits for the cooperation data from siblings. If it successfully receives the data, it returns the ACK directly and advances to step 6 .

Step 6: the parent determines whether it overhears the forwarding or receives ACK from the child, if so, $i=i+1$; otherwise, $i=i$. Step 7: after a transmission cycle, if $i=N_{i}$, then $j=j+1$, if $i<N_{i}$, then $j=j$, and return to step 1 . 


$$
T_{\mathrm{CARQ}}^{o i e}=\sum_{j=1}^{M} \max _{\substack{1<i \leq R \\ M_{i}=j}}\left[E\left(X_{i}\right)\left(p^{N(i)} v(i)+\left(1-p^{N(i)}\right)\left(2 \tau_{b}+\tau_{f}\right)\right)\right] .
$$

\section{Numerical Analysis of Four Collaborative Broadcasting Schemes}

The performances of the four proposed schemes are discussed through numerical analysis and compared with the SW-BHB-ARQ, SW-BHB-oiARQ, and SW-BHB-ieARQ protocol in [3] to verify the superiority of introducing collaborative technology.

For the convenience of description, we refer to SW-BHBCARQ, SW-BHB-oiCARQ, SW-BHB-ieCARQ, SW-BHBoieCARQ, SW-BHB-ARQ, SW-BHB-ARQ, SW-BHB-oiARQ, and SW-BHB-ieARQ protocols as CARQ, oiCARQ, ieCARQ, oieCARQ, ARQ, oiARQ, and ieARQ, respectively.

The parameters of the system are assumed as follows: the range of reliability $\beta$ is $0.9-0.99$, the range of broadcast probability $p$ is $0.3-0.95$, the range of feedback $q$ probability is $0.5-0.95$, the parameter $r$ is equal to 0.7 , broadcasting delay $\tau_{b}=1$, feedback delay $\tau_{f}=\left(\tau_{b} / 4\right)$, and overhearing delay $\tau_{s}=\left(\tau_{b} / 2\right)$.

Figure 9 describes the comparison of maximum retransmission number for CARQ and ARQ protocol based on the values in Table 7. As can be seen from Figure 9, the maximum number of retransmissions for both protocols is equal. This is because that the system reliability only discusses the reliability of root-to-leaf link, regardless of the involvement of sibling collaborations. At the same time, it is obvious that the maximum retransmission number of two protocols both increase with the system reliability.

Figure 10 describes the comparison of maximum retransmissions for CARQ and ARQ protocols under different feedback probabilities based on Table 7. From Figure 10 , it can be seen that the maximum number of retransmissions of two protocols both decrease with the increase of broadcast probability, but it is independent of the feedback probability because the system reliability is only related to the transmission link without the feedback link.

Figures 11 and 12 describe the energy consumption comparison of ARQ and CARQ protocols under different reliability and feedback probabilities based on the values in Table7, respectively.

Figures 11 and 12 show that when the broadcast probability is small, the energy consumption of CARQ is smaller than ARQ, but when the broadcast probability is large, the energy consumption of CARQ is larger than ARQ. Obviously, with the increase of broadcasting probability, the reception probability of terminal in CARQ protocol is greater than ARQ. Meanwhile, the collaboration probability is also greater, which makes the feedback energy of terminal become larger.

Figures 13 and 14 describe the energy consumption comparison of oiARQ and oiCARQ protocols under

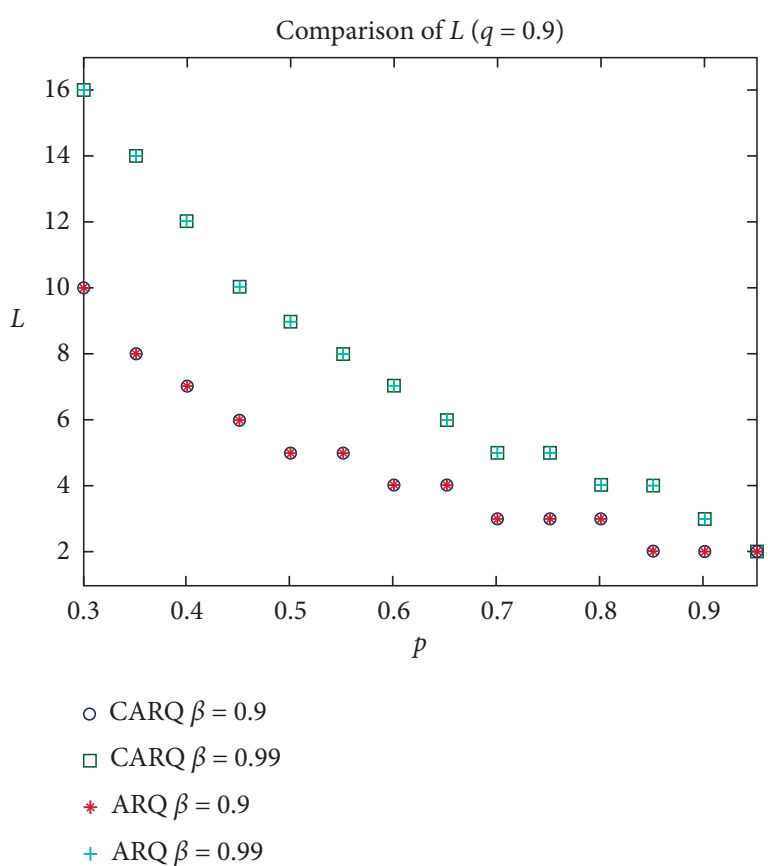

Figure 9: Comparison of $L$ in different $\beta$.

TABLE 7: Energy consumption of ARQ and CARQ protocols.

\begin{tabular}{cccccccc}
\hline & & \multicolumn{6}{c}{$q$} \\
Energy & $p$ & 0.5 & 0.7 & 0.9 & 0.5 & 0.7 & 0.9 \\
& & & $\beta=0.9$ & & & $\beta=0.99$ & \\
\hline \multirow{4}{*}{ ARQ } & 0.3 & 83.61 & 79.10 & 73.66 & 132.55 & 121.86 & 109.23 \\
& 0.6 & 40.08 & 37.45 & 33.84 & 72.95 & 62.76 & 50.38 \\
& 0.9 & 18.60 & 18.60 & 18.60 & 34.20 & 31.00 & 26.09 \\
\hline \multirow{3}{*}{ CARQ } & 0.3 & 92.23 & 78.77 & 62.41 & 138.86 & 107.72 & 75.44 \\
& 0.6 & 46.62 & 39.82 & 30.60 & 78.49 & 55.48 & 34.24 \\
& 0.9 & 19.77 & 19.77 & 19.77 & 35.75 & 31.45 & 24.70 \\
\hline
\end{tabular}

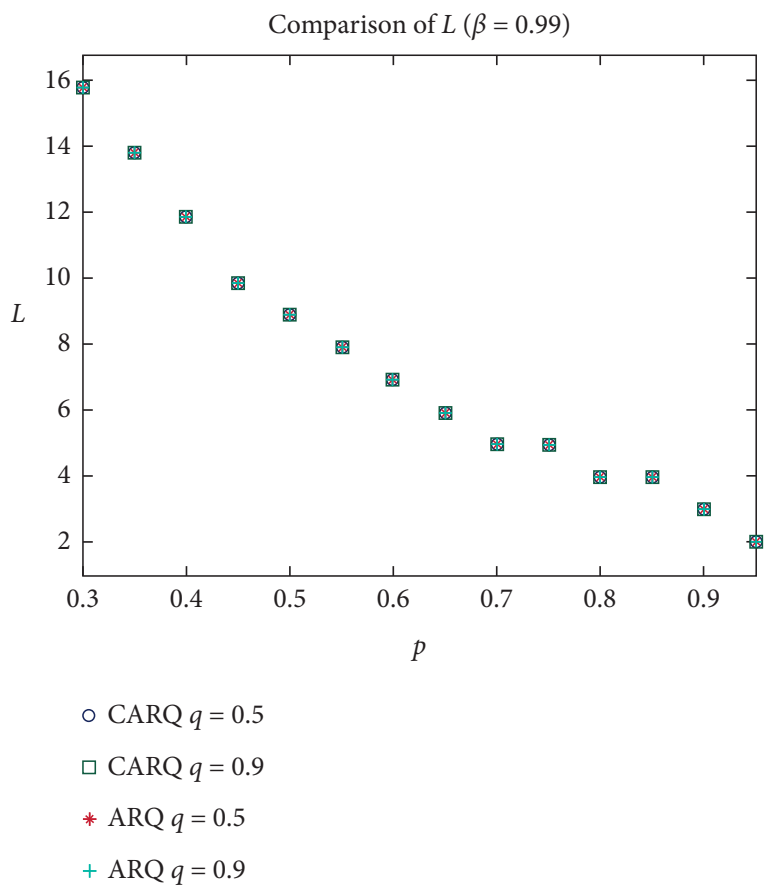

Figure 10: Comparison of $L$ in different $q$. 
Comparison of $E$ under ARQ and CARQ protocols $(q=0.9)$

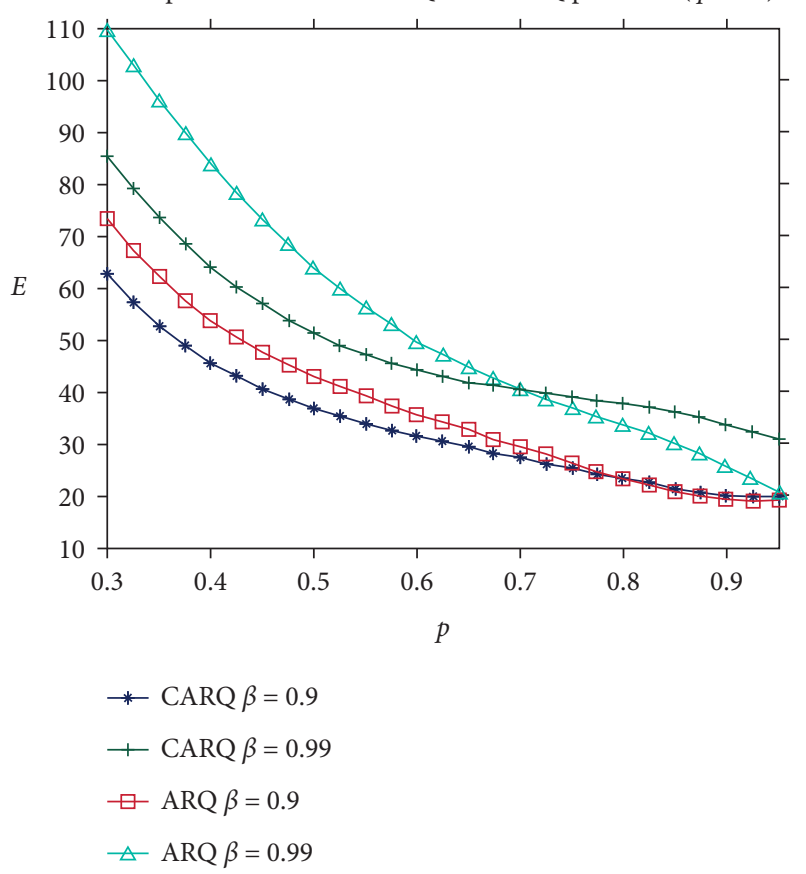

FIgUre 11: Comparison of $E$ of CARQ in different $\beta$.

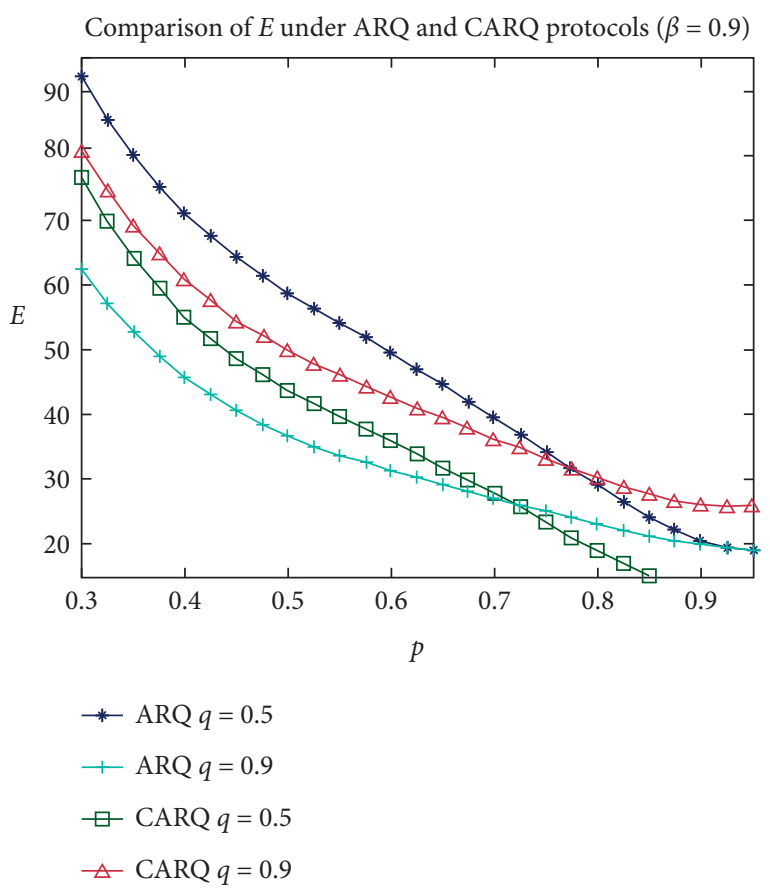

FIgURE 12: Comparison of $E$ of CARQ in different $q$.

different reliability and feedback probabilities based on the values in Table 8, respectively. Unlike ARQ and CARQ protocols, the energy consumption of oiCARQ protocol is always lower than oiARQ because the node broadcast probability increases. Similarly, the energy consumption of oiARQ and oiCARQ protocols is directly proportional to the system reliability and inversely proportional to the feedback probability.

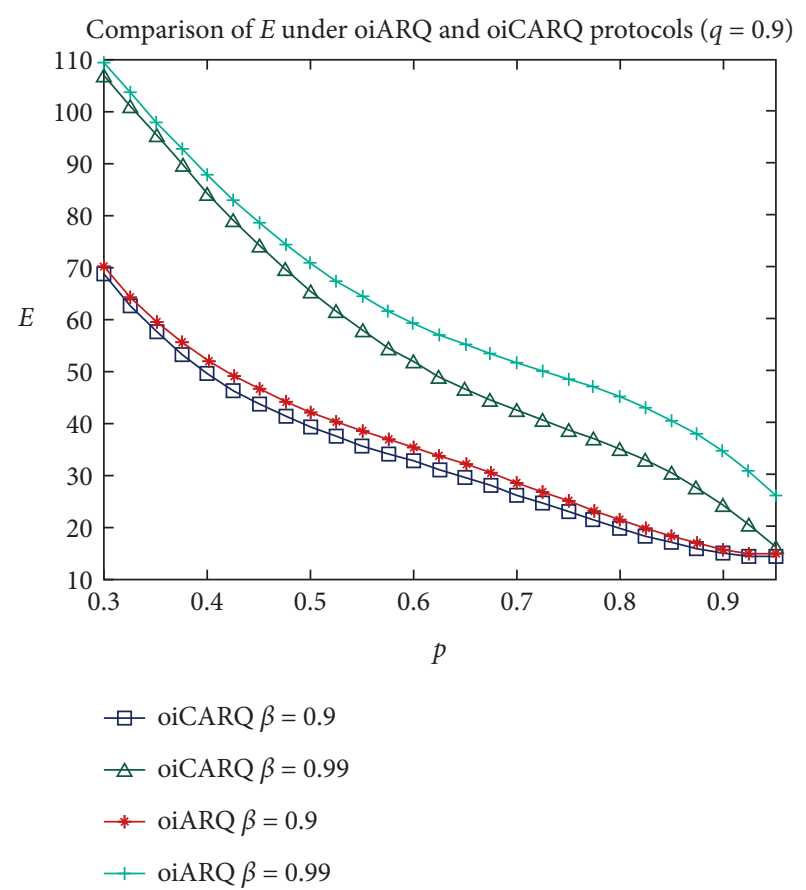

Figure 13: Comparison of $E$ of oiCARQ in different $\beta$.

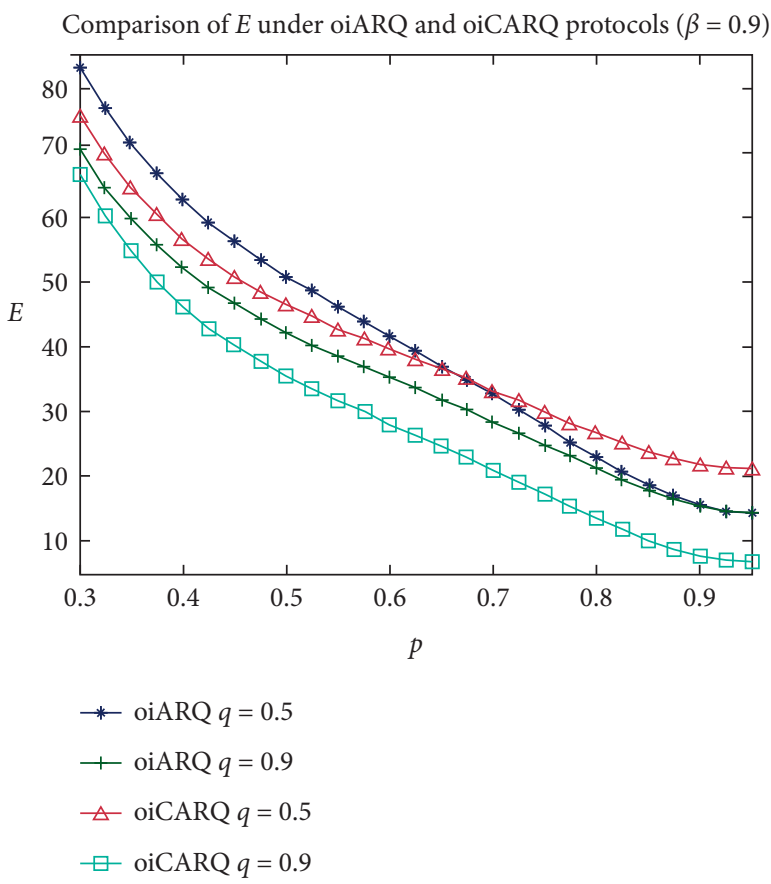

FIgURE 14: Comparison of $E$ of oiCARQ in different $q$.

Figures 15 and 16 describe the comparison of energy consumption of ieARQ and ieCARQ protocols under different reliability and feedback probabilities based on the values in Table 9, respectively. According to the analysis of Section 3.3, the energy consumption of ieCARQ is one constant less than that of CARQ. Therefore, the energy consumption relationship of ieARQ and ieCARQ protocols is the same as that of ARQ and CARQ protocols. In Figure 15, when the system reliability changes from 0.9 to 0.99 , 
TABLE 8: Energy consumption of oiARQ, oiCARQ, and oieCARQ protocols.

\begin{tabular}{lccccccc}
\hline & & \multicolumn{7}{c}{$q$} \\
Energy & $p$ & 0.5 & 0.7 & 0.9 & 0.5 & 0.7 & 0.9 \\
& & & $\beta=0.9$ & & & $\beta=0.99$ & \\
\hline \multirow{3}{*}{ oiARQ } & 0.3 & 73.32 & 71.05 & 68.49 & 117.88 & 112.59 & 106.65 \\
& 0.6 & 32.72 & 31.73 & 30.41 & 61.96 & 57.91 & 52.74 \\
& 0.9 & 17.10 & 14.10 & 14.10 & 27.02 & 26.00 & 24.50 \\
\hline \multirow{3}{*}{ oiCARQ } & 0.3 & 83.02 & 77.43 & 70.09 & 130.94 & 117.00 & 99.62 \\
& 0.6 & 39.05 & 36.72 & 33.33 & 71.95 & 62.38 & 50.15 \\
& 0.9 & 14.97 & 14.97 & 14.97 & 28.54 & 27.21 & 25.19 \\
\hline \multirow{3}{*}{ oieCARQ } & 0.3 & 74.84 & 68.21 & 59.58 & 116.45 & 100.10 & 80.54 \\
& 0.6 & 33.85 & 31.26 & 27.52 & 61.20 & 50.89 & 38.46 \\
& 0.9 & 14.20 & 14.20 & 14.20 & 26.97 & 25.59 & 23.49 \\
\hline
\end{tabular}

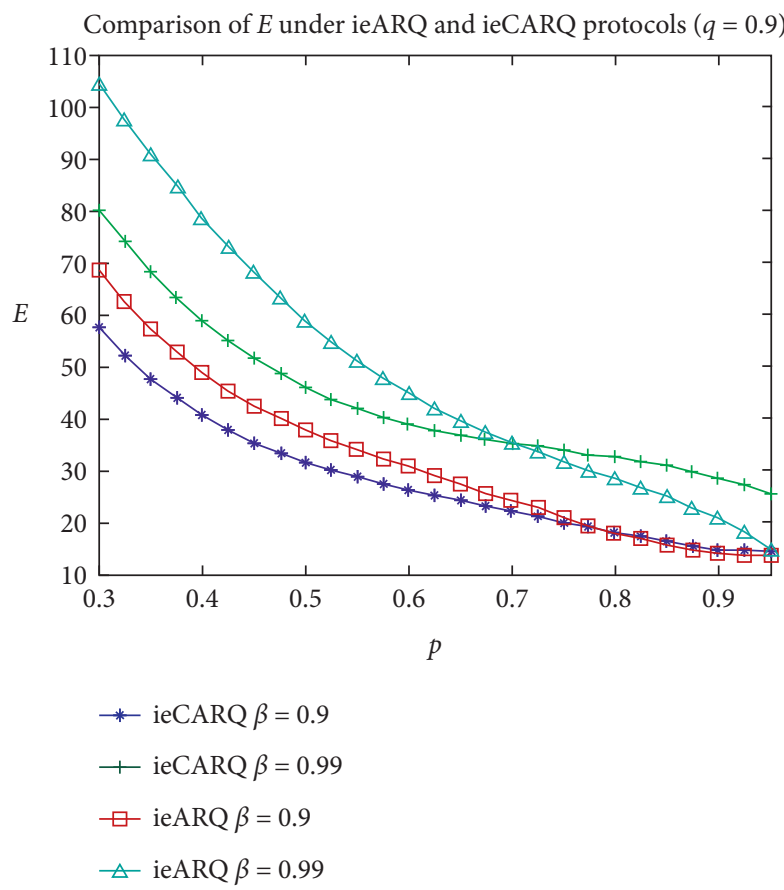

Figure 15: Comparison of $E$ of ieCARQ in different $\beta$.

the critical point of ieCARQ equaling ieARQ decreases from $p=0.75$ to $p=0.68$, which indicates that the higher the reliability, the stricter the broadcast environment where ieCARQ can apply in terms of energy saving.

Figures 17 and 18 describe the energy comparison of four type CARQ broadcast protocols. In Figures 17 and 18, the system energy ranks from small to large as oieCARQ, ieCARQ, oiCARQ, and CARQ. And the greater the reliability, the lower energy of oieCARQ compared with ieCARQ protocol. The lower the feedback probability, the lower energy of oieCARQ compared with ieCARQ protocol. The smaller the broadcast probability, the lower energy of oieCARQ compared with ieCARQ protocol.

Combining these three scenarios, the following conclusions can be drawn. When the communication physical environment is worse, the ieCARQ technology has better advantages in reducing system energy. However, when the communication physical environment is better, the

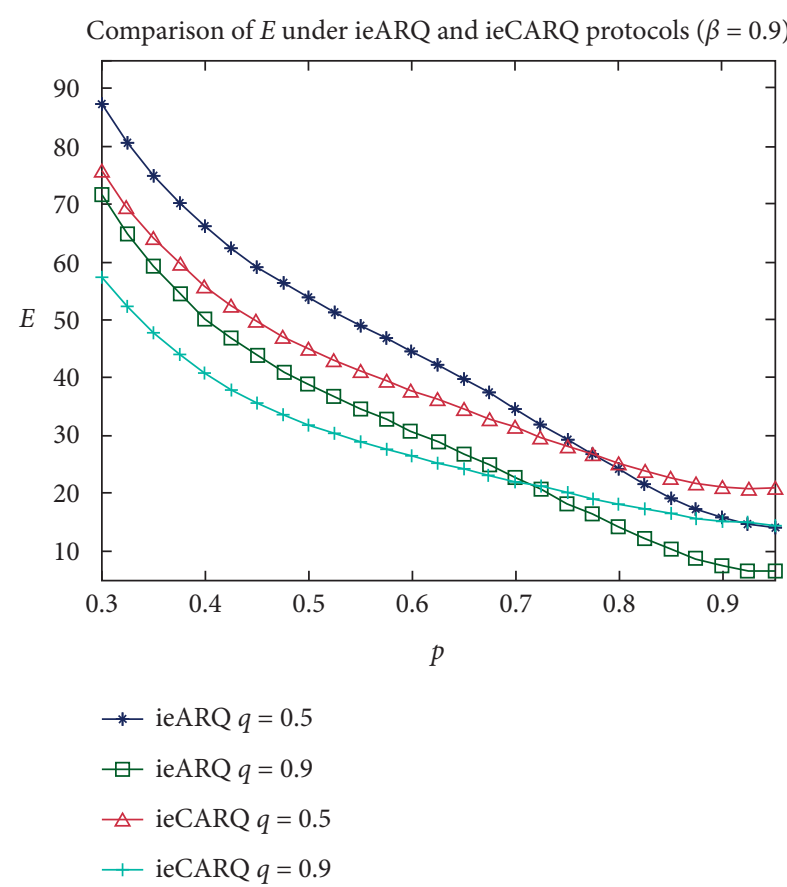

Figure 16: Comparison of $E$ of ieCARQ in different $q$.

TABLE 9: Energy consumption of ieARQ and ieCARQ protocols.

\begin{tabular}{cccccccc}
\hline & & \multicolumn{6}{c}{$q$} \\
Energy & $p$ & 0.5 & 0.7 & 0.9 & 0.5 & 0.7 & 0.9 \\
& & & $\beta=0.9$ & & & $\beta=0.99$ & \\
\hline \multirow{4}{*}{ ieARQ } & 0.3 & 78.61 & 74.10 & 68.66 & 127.55 & 116.86 & 104.23 \\
& 0.6 & 35.08 & 32.45 & 28.84 & 67.95 & 57.76 & 45.38 \\
& 0.9 & 13.60 & 13.60 & 13.60 & 29.20 & 26.00 & 21.09 \\
\hline \multirow{3}{*}{ ieCARQ } & 0.3 & 87.23 & 73.77 & 57.41 & 133.86 & 102.72 & 70.44 \\
& 0.6 & 41.62 & 34.82 & 25.60 & 73.49 & 50.48 & 29.24 \\
& 0.9 & 14.77 & 14.77 & 14.77 & 30.75 & 26.45 & 19.70 \\
\hline
\end{tabular}

differences among oieCARQ, ieCARQ, oiCARQ, and CARQ are very small, so the introduction of collaborative technology is not recommended.

Figures 19 and 20 describe the delay comparison of five types of ARQ/CARQ broadcast protocols under different reliability and feedback probabilities, respectively. Here, the five protocols are ARQ, CAR, oiCARQ, ieCARQ, and oieCARQ protocols. In Figures 19 and 20, the system delay is inversely proportional to broadcast probability and feedback probability and directly proportional to the reliability.

With the same parameters, the system delay is sorted from small to large as ARQ, CARQ, ieCARQ, oieCARQ, and oiCARQ. At the same time, it can be seen that the greater the reliability, the smaller the delay of ARQ than four CARQ protocols, the smaller the feedback probability, and the smaller the delay of ARQ than four CARQ protocols. The smaller the broadcast probability, the smaller the delay of ARQ than four CARQ protocols.

In summary, on the premise of ensuring system reliability, the four proposed CARQ broadcast protocols can effectively reduce energy consumption compared with ARQ protocol. The poorer the physical communication 
Comparison of $E$ under four cooperative broadcast protocols

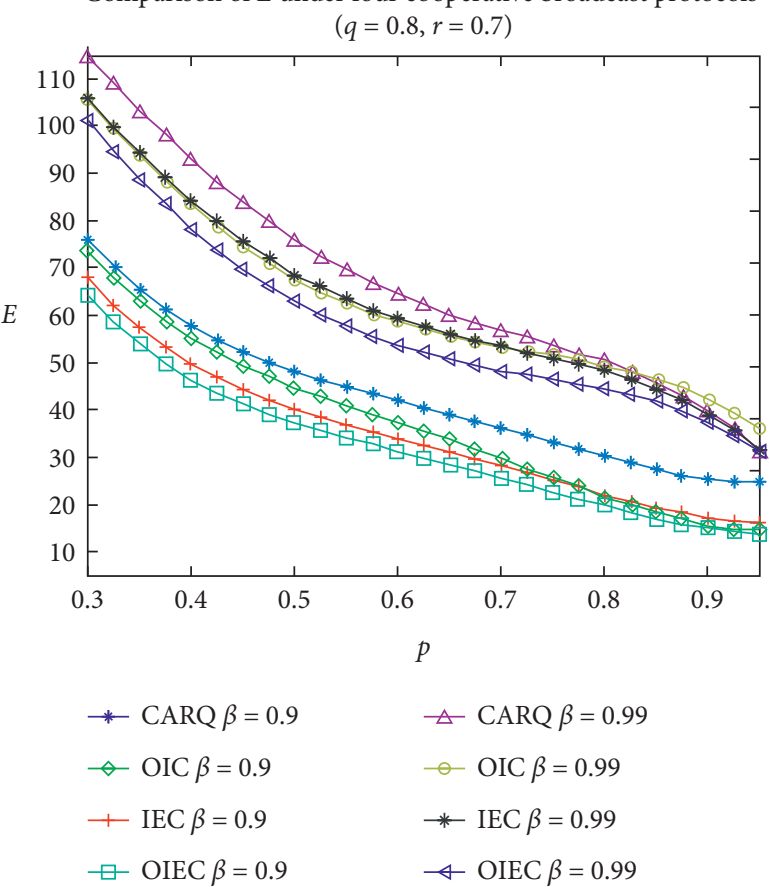

Figure 17: Comparison of $E$ of four cooperative broadcast protocols in different $\beta$.

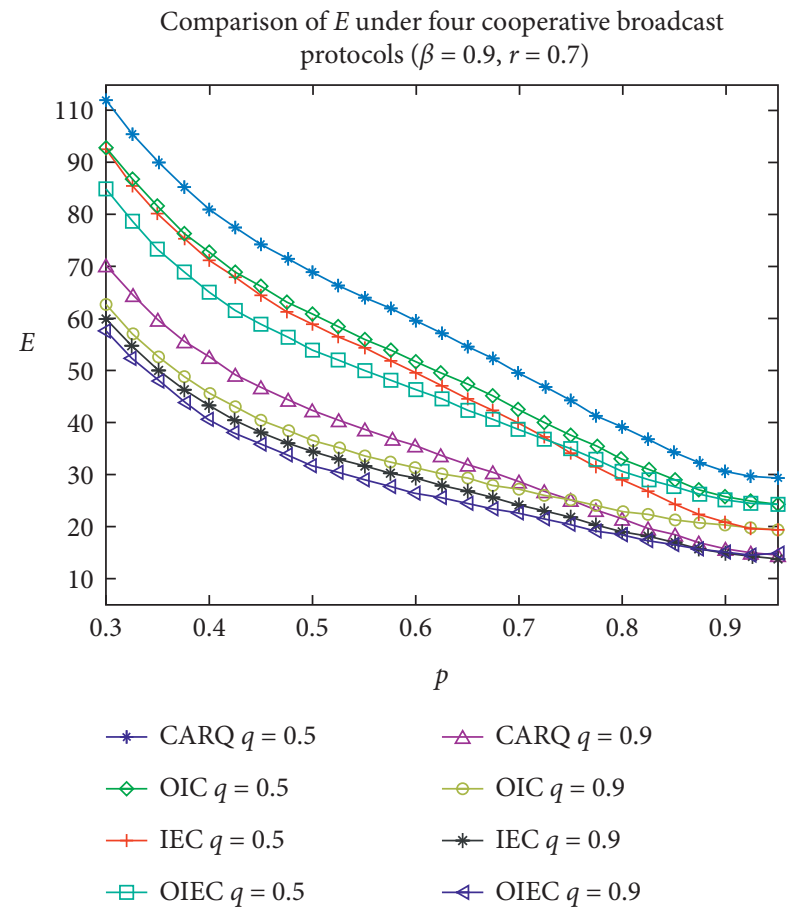

Figure 18: Comparison of $E$ of four cooperative broadcast protocols in different $q$.

environment, the better the energy-saving effect of the CARQ protocols. At the same time, the delay of four proposed CARQ broadcast protocols is much larger than that of

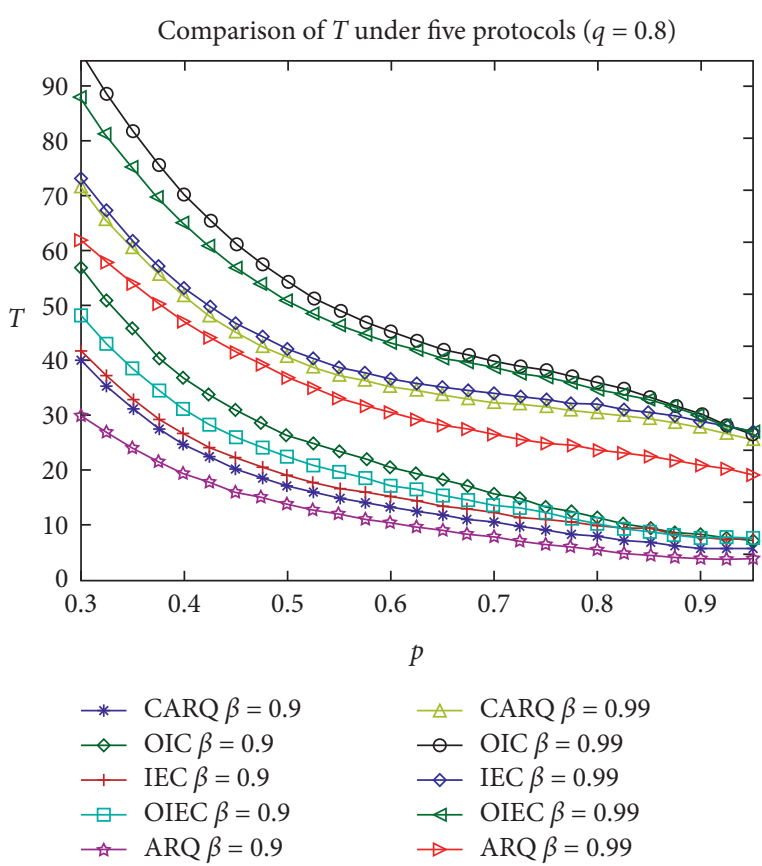

Figure 19: Comparison of $T$ of five protocols in differ $\beta$.

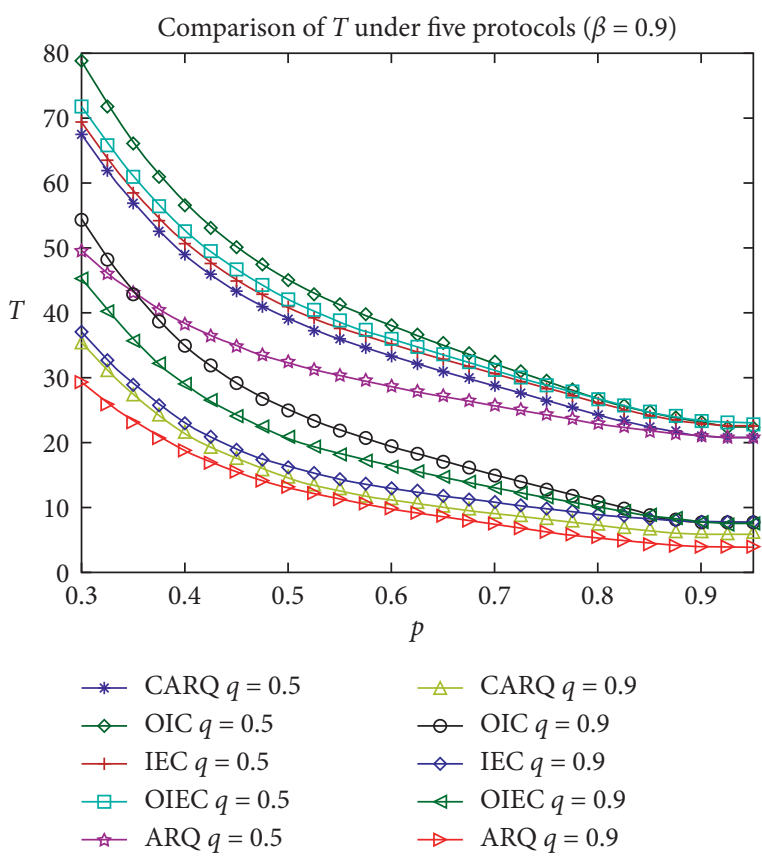

FIgure 20: Comparison of $T$ of five protocols in differ $q$.

the ARQ protocol. The worse the physical communication environment, the longer the delay of the CARQ protocols.

Taking above conclusion to VANET, it is recommended to select different broadcast protocols for different design objectives. At the same time, for the conflicting between delay and energy consumption, how to further select or optimize the cooperative broadcasting scheme to balance this conflicting is our next work. 


\section{Conclusions}

Considering the broadcasting characteristics of radio waves, a cooperative communication technology is introduced to extend the tree-based network to the general mesh network. Four CARQ broadcast protocols, SW-HBH-CARQ, SWHBH-oiCARQ, SW-HBH-ieCARQ, and SW-HBH-oieCARQ, are proposed in this paper. Quantitative analysis of retransmission number, delay, energy consumption, and other performance of the four protocols are also performed. First, under the mesh network, based on energy-saving considerations, the maximum retransmission number satisfying system reliability is calculated to minimize energy consumption. Secondly, through the study of broadcasting characteristics, an analytic formula for the energy consumption and delay are obtained. Finally, the numerical relationships among the maximum retransmission number, energy consumption, delay and reliability, broadcast probability, and feedback probability are discussed. We get the following conclusions: under the four CARQ broadcasting protocols, the energy and delay are inversely proportional to the broadcast probability and feedback probability and directly proportional to the reliability. In order to verify the superiority of collaborative technology, SW-HBH-CARQ, SW-HBH-oiCARQ, and SW-HBH-ieCARQ were compared with the corresponding noncooperative protocols. It can be seen that four CARQ broadcasting protocols reduce system energy but increase delay. For the design of the broadcasting system, the introduction of SW-HBH-oieCARQ technology can effectively reduce energy in the time-delay tolerant network when the communication physical environment is poor. In the network with better communication physical environment, the ARQ broadcasting protocol can meet better system requirements without using collaborative technology.

\section{Data Availability}

The data used to support the findings of this study are available from the corresponding author upon request.

\section{Conflicts of Interest}

The authors declare no conflicts of interest.

\section{Authors' Contributions}

Zufang Dou was responsible for conceptualization, methodology, formal analysis, and writing-original draft preparation. Software was developed by Jianwen Tian; validation was carried out by Liben Yang and Jianwen Tian; investigation was done by Qiaoli Yang. Zufang Dou and Qiaoli Yang were involved in writing-review and editing. All the authors have read and agreed to the published version of the manuscript.

\section{Acknowledgments}

This project was supported by Lanzhou Jiaotong University scientific research fund (grant no. 1520020420), Natural
Science Foundation of Gansu Province of China (grant nos. 20JR5RA428 and 21JR1RA235), and Universities Scientific Research Project of Gansu Province Education Department of China (grant no. 2019A-042).

\section{References}

[1] X. Shen, X. Cheng, L. Yang, R. Zhang, and B. Jiao, "Data dissemination in VANETs: a scheduling approach," IEEE Transactions on Intelligent Transportation Systems, vol. 15, no. 5, pp. 2213-2223, 2014.

[2] K. Zheng, Q. Zheng, P. Chatzimisios, W. Xiang, and Y. Zhou, "Heterogeneous vehicular networking: a survey on architecture, challenges, and solutions," IEEE Communications Surveys \& Tutorials, vol. 17, no. 4, pp. 2377-2396, 2015.

[3] S. Li, Z. Dou, F. Wang, and Q. Xu, "Energy efficiency of five broadcast-based ARQ protocols in multi-hop wireless sensor networks," IET Communications, vol. 13, no. 15, pp. 2243-2253, 2019.

[4] F. J. Ros, P. M. Ruiz, and I. Stojmenovic, "Acknowledgmentbased broadcast protocol for reliable and efficient data dissemination in vehicular ad hoc networks," IEEE Transactions on Mobile Computing, vol. 11, no. 1, pp. 33-46, 2012.

[5] O. Rehman, M. Ould-Khaoua, and H. Bourdoucen, "An adaptive relay nodes selection scheme for multi-hop broadcast in VANETs," Computer Communications, vol. 87, pp. 76-90, 2016.

[6] H. Yoo and D. Kim, "ROFF: robust and fast forwarding in vehicular ad-hoc networks," IEEE Transactions on Mobile Computing, vol. 14, no. 7, pp. 1490-1502, 2015.

[7] C. Suthaputchakun, M. Dianati, and Z. Sun, "Trinary partitioned black-burst-based broadcast protocol for time-critical emergency message dissemination in VANETs," IEEE Transactions on Vehicular Technology, vol. 63, no. 6, pp. 2926-2940, 2014.

[8] Q. Qing Xu, T. Mak, J. Jeff Ko, and R. Sengupta, “Medium access control protocol design for vehicle-vehicle safety messages," IEEE Transactions on Vehicular Technology, vol. 56, no. 2, pp. 499-518, 2007.

[9] A. Naja, M. Boulmalf, and M. Essaaidi, "A distributed priority-based rebroadcasting protocol for VANETs: mitigating the storm problem," Mobile Networks and Applications, vol. 24, no. 5, pp. 1555-1568, 2019.

[10] J. Yang, C. Huang, and X. Fan, "Reliable broadcast mechanism for emergency message in urban vehicular ad hoc networks," IET Intelligent Transport Systems, vol. 13, no. 9, pp. 1383-1393, 2019.

[11] K. Satheshkumar and S. Mangai, "EE-FMDRP: EE-FMDRP: energy efficient-fast message distribution routing protocol for vehicular ad-hoc networks," Journal of Ambient Intelligence and Humanized Computing, vol. 12, no. 3, pp. 3877-3888, 2020.

[12] K. K. Rana, S. Tripathi, and R. S. Raw, "Opportunistic directional location aided routing protocol for vehicular ad-hoc network," Wireless Personal Communications, vol. 110, no. 3, pp. 1217-1235, 2020.

[13] S. Kad and V. K. Banga, "A position based speed adaptive dissemination approach for vehicular ad hoc networks," Wireless Personal Communications, vol. 115, no. 2, pp. 1783-1804, 2020.

[14] Y. Bi, H. Shan, X. S. Shen, N. Wang, and H. Zhao, "A multihop broadcast protocol for emergency message dissemination in urban vehicular ad hoc networks," IEEE Transactions on 
Intelligent Transportation Systems, vol. 17, no. 3, pp. 736-750, 2016.

[15] S. Ucar, S. C. Ergen, and O. Ozkasap, "Multihop-cluster-based IEEE $802.11 \mathrm{p}$ and LTE hybrid architecture for VANET safety message dissemination," IEEE Transactions on Vehicular Technology, vol. 65, p. 1, 2015.

[16] Y. Mylonas, M. Lestas, A. Pitsillides, P. Ioannou, and V. Papadopoulou, "Speed adaptive probabilistic flooding for vehicular ad hoc networks," IEEE Transactions on Vehicular Technology, vol. 64, no. 5, pp. 1973-1990, 2015.

[17] R. Tizvar and M. Abbaspour, "A density-aware probabilistic interest forwarding method for content-centric vehicular networks," Vehicular Communications, vol. 23, pp. 1-11, 2020.

[18] S. Li, Z. Dou, F. Wang, and Q. Xu, "Energy efficiency of five broadcast-based ARQ protocols in multi-hop WSNs," IET Communications, vol. 13, pp. 2243-2253, 2019.

[19] A. Srivastava, A. Prakash, and R. Tripathi, "Fuzzy-based beaconless probabilistic broadcasting for information dissemination in urban VANET," Ad Hoc Networks, vol. 108, pp. 1-18, 2020.

[20] C. Borrego, J. Borrell, and S. Robles, "Efficient broadcast in opportunistic networks using optimal stopping theory," Ad Hoc Networks, vol. 88, pp. 5-17, 2019.

[21] A. Tomes, T. C. Carlos, J. C. Cano et al., "Evaluation of flooding schemes for real-time video transmission in VANET," Ad Hoc Networks, vol. 24, no. PB, pp. 3-20, 2015.

[22] M. B. Yassein, S. F. Nimer, and A. Y. Al-Dubai, "A new dynamic counter-based broadcasting scheme for mobile ad hoc networks," Simulation Modelling Practice and Theory, vol. 19, pp. 553-563, 2011.

[23] A. Naja, M. Essaaidi, and M. Boulmalf, "CPROB: a dynamic hybrid broadcasting protocol for vehicular ad hoc networks," in Proceedings of the 2016 International Conference Electrical and Information Technologies (ICEIT), pp. 355-361, Tangiers, Morocco, May 2016.

[24] M. Bakhouya, J. Gaber, and P. Lorenz, "An adaptive approach for information dissemination in vehicular ad hoc networks," Journal of Network and Computer Applications, vol. 34, no. 6, pp. 1971-1978, 2011.

[25] E. Limouchi and I. Mahgoub, "Volunteers Dilemma game inspired broadcast scheme for vehicular ad hoc networks," IEEE Transactions on Intelligent Transportation Systems, vol. 20, no. 12, pp. 4439-4449, 2019.

[26] P.-W. Tsai, J.-P. Li, J.-S. Shih et al., "Mixed broadcast techniques of leisure information in vehicular ad-hoc networks," Telecommunication Systems, vol. 75, no. 2, pp. 221-234, 2016.

[27] J. Kim, S. W. Choi, W.-Y. Shin, Y.-S. Song, and Y.-K. Kim, "Group communication over LTE: a radio access perspective," IEEE Communications Magazine, vol. 54, no. 4, pp. 16-23, 2016.

[28] M. Ali, A. W. Malik, A. U. Rahman, S. Iqbal, and M. M. Hamayun, "Position-based emergency message dissemination for Internet of vehicles," International Journal of Distributed Sensor Networks, vol. 15, no. 7, pp. 1-12, 2019.

[29] W. Benrhaiem, A. Hafid, and P. K. Sahu, "Reliable emergency message dissemination scheme for urban vehicular networks," IEEE Transactions on Intelligent Transportation Systems, vol. 21, no. 3, pp. 1154-1166, 2020.

[30] Z. Rosberg, R. P. Liu, T. L. Dinh, Y. F. Dong, and S. Jha, "Statistical reliability for energy efficient data transport in wireless sensor networks," Wireless Networks, vol. 16, no. 7, pp. 1913-1927, 2010.

[31] Z. Dou, S. Li, Y. Gao, and J. Chen, "Energy efficiency of CARQ broadcast protocol," in Proceedings of the 2018 IEEE 3rd
Advanced Information Technology, Electronic and Automation Control Conference (IAEAC 2018), pp. 10.12-10.14, ChongQing, China, October 2018. 